\title{
Simulations of extensional flow in microrheometric devices
}

\author{
Mónica S. N. Oliveira · Lucy E. Rodd · \\ Gareth H. McKinley · Manuel A. Alves
}

Received: 21 June 2007/ Accepted: 7 January 2008/Published online: 15 April 2008

(C) Springer-Verlag 2008

\begin{abstract}
We present a detailed numerical study of the flow of a Newtonian fluid through microrheometric devices featuring a sudden contraction-expansion. This flow configuration is typically used to generate extensional deformations and high strain rates. The excess pressure drop resulting from the converging and diverging flow is an important dynamic measure to quantify if the device is intended to be used as a microfluidic extensional rheometer. To explore this idea, we examine the effect of the contraction length, aspect ratio and Reynolds number on the flow kinematics and resulting pressure field. Analysis of the computed velocity and pressure fields show that, for typical experimental conditions used in microfluidic devices, the
\end{abstract}

Electronic supplementary material The online version of this article (doi:10.1007/s10404-008-0277-5) contains supplementary material, which is available to authorized users.

M. S. N. Oliveira · M. A. Alves $(\bowtie)$

CEFT, Departamento de Engenharia Química,

Faculdade de Engenharia da Universidade do Porto,

Rua Dr. Roberto Frias, 4200-465 Porto, Portugal

e-mail: mmalves@fe.up.pt

M. S. N. Oliveira

e-mail: monica.oliveira@fe.up.pt

L. E. Rodd · G. H. McKinley

Department of Mechanical Engineering, MIT,

77 Massachusetts Avenue, Cambridge, MA 02139-4307, USA

e-mail: 1.rodd@uq.edu.au

G. H. McKinley

e-mail: gareth@mit.edu

L. E. Rodd

Department of Chemical and Biomolecular Engineering,

The University of Melbourne, Melbourne, VIC 3010, Australia steady flow is highly three-dimensional with open spiraling vortical structures in the stagnant corner regions. The numerical simulations of the local kinematics and global pressure drop are in good agreement with experimental results. The device aspect ratio is shown to have a strong impact on the flow and consequently on the excess pressure drop, which is quantified in terms of the dimensionless Couette and Bagley correction factors. We suggest an approach for calculating the Bagley correction which may be especially appropriate for planar microchannels.

Keywords Microfluidics - Microrheometry · Couette correction - Bagley correction .

Contraction-expansion flow $\cdot$ Extensional flow

\section{Introduction}

Over the past decade, microfluidic devices have emerged as a powerful toolset for miniaturization and automation of fluid handling and fluid analysis (Whitesides 2006), significantly reducing the time and cost involved in diagnostic procedures. The numerous advantages of microfluidics, namely the reduced amounts of sample and reagents needed, the high surface-to-volume ratio, the substantial waste reduction, the low cost of fabrication and the possibility of producing highly integrated and disposable devices, have stimulated remarkable interest and unraveled an extensive range of applications. As a result of the fabrication methods employed, micro-devices are typically composed of a series of planar channels and many of the geometries used involve changes in the cross-sectional flow area (Lee et al. 2002). For this reason, understanding the fluid dynamics in prototypical configurations, such as the planar contractionexpansion, is of utmost importance. 
Viscous flow through contractions (planar and axisymmetric) is a long-standing numerical benchmark problem, particularly for viscoelastic fluids (Hassager 1988; Brown and McKinley 1994; Owens and Phillips 2002). This type of flow results in streamwise acceleration and is of particular importance in polymer processing applications, such as injection molding, spinning and film blowing (Barnes et al. 1989). The flow through sudden contractions, including the complex effects of flow geometry and fluid rheology on the flow characteristics, has been the subject of thorough reviews by Boger (1987) and White et al. (1987). More recent results (numerical and experimental) can be found in the book by Owens and Phillips (2002) and the introductions of Alves et al. (2005), Rodd et al. (2005) and Oliveira et al. (2007b).

The ultimate goal of this research is to develop an efficient microfluidic "rheometer-on-a-chip" capable of achieving high strain rates, in such a way that it can be used to measure the effective extensional properties of dilute polymeric solutions and low viscosity complex fluids (e.g. inks, coating fluids, DNA solutions, blood). In this paper we investigate the flow of a Newtonian fluid (distilled water) through a microfabricated geometry containing a planar contraction-expansion section. We combine experimental measurements with numerical computations to obtain accurate velocity profiles, flow patterns and recirculation sizes as well as pressure drop results. As we show below, excellent agreement can be obtained between the experimental and numerical techniques, validating the use of future numerical computations as an exploratory design tool. The present study is a starting point for a more indepth experimental and numerical study of the flow of viscoelastic fluids in these types of micro-geometries. However, as we shall demonstrate, even for a simple Newtonian fluid the flow in such a device can be complex and is yet to be completely understood.

\subsection{Fluid physics of converging entry flows}

Even though the geometry is simple, converging entry flows show complex flow patterns that feature both shearing and elongational regions: near the walls the flow is shear-dominated, whereas along the centerline it is predominantly extensional and essentially shear-free (Rothstein and McKinley 2001; Alves and Poole 2007). In this geometry the flow can exhibit quite complex behavior, even for laminar flows of Newtonian fluids (e.g. Fearn et al. 1990; Hawa and Rusak 2001; Mishra and Jayaraman 2002; Oliveira 2003) resulting in either symmetric or asymmetric flow patterns downstream of the expansion plane, depending on the value of the Reynolds number and on the geometrical characteristics of the device (Battaglia et al. 1997).

At the microscale, entry flows are also important in many industrial applications, ranging from inkjet printing to micro-injection molding. These processes usually involve a strong extensional component: for example, inkjet printing, which is a high volume commercial industry, depends on the extensional flow of slightly elastic non-Newtonian fluids at the microscale (Oliveira et al. 2006; Tuladhar and Mackley 2008). The small length-scales of the geometries usually result in a range of Reynolds numbers well within the laminar flow regime. Furthermore, entry flows at the microscale are typically characterized by large contraction ratios, and thus both the extensional deformation rate and the total extensional strain are high. Consequently, a large additional pressure drop (or "extra pressure drop") at the converging entrance is observed, even for Newtonian fluids, and this can be related to the extensional properties of the solution (Kang et al. 2005). A microfluidic geometry consisting of an abrupt contraction followed by an abrupt expansion may thus be used in order to determine an apparent extensional viscosity of dilute polymer solutions from pressure drop measurements in micro-contractions (Rodd et al. 2005). The underlying idea is to use streamwise acceleration of the fluid in the throat in order to achieve a local planar elongational flow in the core of the converging section, such that the velocity increases linearly toward the throat. If the contraction-expansion geometry is abrupt, spatially inhomogeneous extensional behavior is observed in the entrance region, and this can make it difficult to identify a region of constant strain rate. The resulting material coefficient is therefore most accurately described as an "apparent extensional viscosity" (Rodd et al. 2005). Nonetheless, these microfluidic contraction-expansion flows are extensionally dominated and therefore important information can still be extracted from differential pressure measurements in a similar fashion to that performed in conventional contraction geometries (Cogswell 1972a, b; James et al. 1990; Binding et al. 1998).

At the macroscale, the major flow pattern typical of Newtonian fluid flow through planar contraction geometries is the formation of an upstream Moffatt vortex (Moffatt 1964), close to the corner, which gradually diminishes as the effects of fluid inertia increase. For Newtonian fluids, the most interesting dynamical features of this flow take place in the expansion region. Early experimental studies of laminar flows through macroscale planar channels with a sudden expansion (Durst et al. 1974; Cherdron et al. 1978), revealed the existence of two recirculation regions of equal size downstream of the expansion for low Reynolds number flows. As the Reynolds number is increased, symmetry about the centerline is initially maintained and the recirculation length increases progressively (Durst et al. 1974; Cherdron et al. 1978). However, above a certain value of the Reynolds number, a supercritical bifurcation is observed and one recirculation zone expands while the other shrinks as the Reynolds number is further increased (Drikakis 1997; 
Revuelta 2005). Despite this asymmetry, the flow remains temporally steady for a certain range of Reynolds number (Mishra and Jayaraman 2002). This phenomenon, in which stable asymmetric vortices are formed, is seen in 2D planar expansions and is frequently attributed to the Coanda effect (Wille and Fernholz 1965). Infinitesimal perturbations of the velocity field cause the flow to be shifted to one side of the expansion, generating a velocity increase near one wall and a corresponding pressure decrease near that same wall. As a result, the flow asymmetry is likely to be maintained once the resulting pressure difference is established. The pattern of vortex development and growth has been observed experimentally and confirmed by a series of numerical studies. The numerical computations of Sobey and Drazin (1986) and Fearn et al. (1990) together with the linear stability analysis of Shapira et al. (1990) indicate that this behavior occurs as a result of a bifurcation in the solution of the Navier-Stokes equations, i.e. above a critical Reynolds number two symmetric stable solutions co-exist (Battaglia et al. 1997). Hawa and Rusak (2001) explain the loss of symmetric stability as a result of the interaction between the effects of viscous dissipation, the downstream convection of perturbations by the base symmetric flow, and the upstream convection induced by $2 \mathrm{D}$ asymmetric disturbances.

Oliveira (2003) predicted that, for Newtonian fluids in a 1:3 planar 2D expansion, the flow becomes asymmetric above a critical Reynolds number of $R e_{\mathrm{c}} \approx 54$. This result is in good agreement with previous works (Shapira et al. 1990; Fearn et al. 1990; Drikakis 1997; Mishra and Jayaraman 2002). However this critical Reynolds number is found to depend strongly on the expansion ratio, $w_{\mathrm{d}} / w_{\mathrm{c}}$, where $w_{\mathrm{c}}$ and $w_{\mathrm{d}}$ are the small and large channel widths, respectively (Drikakis 1997; Revuelta 2005). Drikakis (1997) performed a numerical study of the laminar flow through sudden expansions with varying expansion ratios and found that the critical Reynolds number decreases with increasing expansion ratio and that the asymmetries become stronger with increasing Reynolds number. Furthermore, in 3D geometries, the critical Reynolds number varies with the aspect ratio, $\mathrm{AR}=h / w_{\mathrm{c}}$, where $h$ is the depth of the channel in the "neutral" direction (Cherdron et al. 1978; Chiang et al. 2000).

An important difference in nomenclature must be noted here: at macroscales, a "planar geometry" commonly refers to flow cells with large aspect ratios $(A R>>1)$ which are commonly used to approximate a 2D planar flow, i.e. the depth of the channel is designed to be large $\left(h / w_{\mathrm{c}}>>1\right)$ so that the effects of the endwalls are negligible. When the aspect ratio decreases, the effects of the endwalls increase; in expansion flows, for example, the critical Reynolds number for the bifurcation increases, and eventually the flow patterns observed experimentally no longer become asymmetric at large Reynolds numbers (Chiang et al. 2000). For moderate aspect ratios, the flow is steady in time but highly three-dimensional, and complex spiraling structures are observed, which are not closed recirculating cells as in the case of ideal 2D expansion flows. By contrast, in lithographic microfabrication techniques, the term "planar geometry" typically refers to devices in which the depth, $h$, of the flow channel is kept constant throughout the device and variations to the cross-sectional flow area (such as a contraction and/or expansion) occur only in the flow direction. This typically leads to aspect ratios close to unity in the contraction region ( $\mathrm{AR}=$ $\left.h / w_{\mathrm{c}}=O(1)\right)$ and much smaller aspect ratios upstream and downstream of the region of interest $\left(h / w_{\mathrm{d}}<<1\right)$. Steady viscous flow in microfluidic devices should thus be expected to be three-dimensional in nature. Tsai et al. (2006) examined numerically the flow of Newtonian fluids through microfabricated planar expansions and found that the flow only resembles a 2D flow for very high aspect ratios ( $A R>>1$ ); otherwise near the expansion plane the flow is locally 3D. The theoretical study of Lauga et al. (2004) shows that even for low aspect ratios $(A R<1)$ the flow is highly three-dimensional, a result that is more important whenever there are abrupt changes in the crosssection of the geometry.

The pressure drop is an important dynamical quantity associated with viscous flow through channels having sudden changes in cross-sectional area. In entry flows, the total pressure drop is composed of two parts, one caused by the pressure drop along the length of the channel due to fully developed viscous flow plus an excess pressure drop that arises from the entrance and exit effects. The latter component results from streamwise deformations associated with characteristic features like contractions and expansions and may represent a significant portion of the total pressure drop, even in the case of Newtonian fluid flows (Kang et al. 2005, 2006; Oliveira et al. 2007a), particularly when a short contraction channel is used. This extra pressure drop is difficult to measure directly by experimental means (Kang et al. 2006) and is usually presented in terms of a dimensionless Couette correction or a Bagley correction (Bagley 1957; Boger 1982) obtained by extrapolating experimental measurements. These "end effects" originate mainly from the streamwise rearrangement of the velocity profile and the resulting extensional flow (Kang et al. 2006), therefore the excess pressure drop yields information about the global state of viscoelastic stresses in the flow (Rothstein and McKinley 2001). In fact, many researchers have attempted to use the excess pressure as a means to estimate the extensional viscosity of viscoelastic fluids and approximate analyses are often used (Cogswell 1972a; Binding et al. 1988; Boger and Binnington 1990; James et al. 1990; Rothstein and McKinley 2001; Rodd et al. 2005). 
The paper is organized as follows. In Sect. 2, we describe the experimental geometry, method of fabrication and the experimental techniques used for the measurements. In Sect 3, the governing equations and the numerical method used to solve them are summarized. The flow geometry and the computational meshes used are also outlined. The experimental and numerical results are discussed in Sects. 4-6: the global flow field and the characteristics of the recirculations are analyzed in Sect. 4; in Sect. 5 a detailed study of the velocity field is carried out; and in Sect. 6 the corresponding pressure drops are evaluated. Finally, in Sect. 7 we summarize the main conclusions of this work.

\section{Experimental}

\subsection{Channel geometry and fabrication}

The channels were fabricated in polydimethylsiloxane (PDMS) from an SU-8 photoresist mold using standard soft-lithography techniques (McDonald et al. 2000). The master molds were prepared using a high-resolution chrome mask together with a contrast enhancer and a barrier coat to allow for smooth, nearly vertical walls and sharp, well-defined corner features. Each set of PDMS channels was bonded permanently to a microscope cover slip after being air plasma treated. A detailed description of the experimental channel fabrication outlined above can be found in Rodd et al. (2005).

A sketch of the micro-geometry used in this work is presented in Fig. 1. The set of channels was designed to have approximately a 16:1:16 contraction-expansion ratio and different contraction lengths were tested: $L_{\mathrm{c}}=100$, 200 and $400 \mu \mathrm{m}$. In all cases, the channel depth, $h=55 \mu \mathrm{m}$, the width of the upstream and downstream channels, $w_{\mathrm{u}}=w_{\mathrm{d}}=400 \mu \mathrm{m}$, and the contraction width were kept constant. The mask used to produce the channels resulted in a final contraction width of $w_{\mathrm{c}}=26 \mu \mathrm{m}$ (as determined by profilometry) instead of the targeted $25 \mu \mathrm{m}$ required to achieve the 16:1:16 contractionexpansion ratio; so in effect we have a 15.4:1:15.4 ratio. The point located at the centerline of the contraction plane and midway between each end-wall is taken as the coordinate origin $(x=y=z=0)$. The length of the upstream and downstream channels is long enough that the flow reaches a fully developed velocity profile at all flow rates studied.

Pressure taps were located $3 \mathrm{~mm}$ upstream and downstream of the contraction plane $(z=0)$. A constant displacement-rate syringe pump was used to impose a constant flow rate into the micro-device $10 \mathrm{~mm}$ upstream of the contraction plane, over a wide range of flow rates $0.1 \leq Q \leq 8 \mathrm{ml} / \mathrm{h}$. A brief description of the experimental configuration and procedures is given below; further details can be found in Rodd et al. (2005).

\subsection{Measurement techniques}

The steady flow kinematics were characterized using fluorescent streak imaging and micro-particle image velocimetry $(\mu \mathrm{PIV})$. For the streak imaging, the fluid was seeded with $1.1 \mu \mathrm{m}$ diameter fluorescent particles (Ex/ $\mathrm{Em}=520 / 580 \mathrm{~nm}$ ) at a weight concentration of $0.02 \%$. A CCD camera $(2,184 \times 1,472$ pixels $)$ with a $30 \mathrm{~ms}$ exposure time was used to acquire the images. This camera was connected to an inverted microscope with an epi-fluorescence attachment and a $10 \mathrm{X}$ objective with numerical aperture $\mathrm{NA}=0.3$. The flow was illuminated with a mercury light in combination with a dichroic mirror and filter set in order to isolate the $546 \mathrm{~nm}$ peak of the mercury lamp light spectrum, and the resultant particle fluorescence signal $(580 \mathrm{~nm})$. For the set-up used the depth of measurement corresponds to $29.7 \mu \mathrm{m}$, which amounts to $54 \%$ of the channel depth in the observable streak line images (Rodd et al. 2005).

Fig. 1 Optical transmission microscope image and schematic diagram of the 400:26:400 abrupt planar contraction-expansion showing the coordinate system and variables used throughout the text. The contraction ratio is defined as $\mathrm{CR}=w_{\mathrm{u}} / w_{\mathrm{c}}$ and the aspect ratio as $\mathrm{AR}=h / w_{\mathrm{c}}$
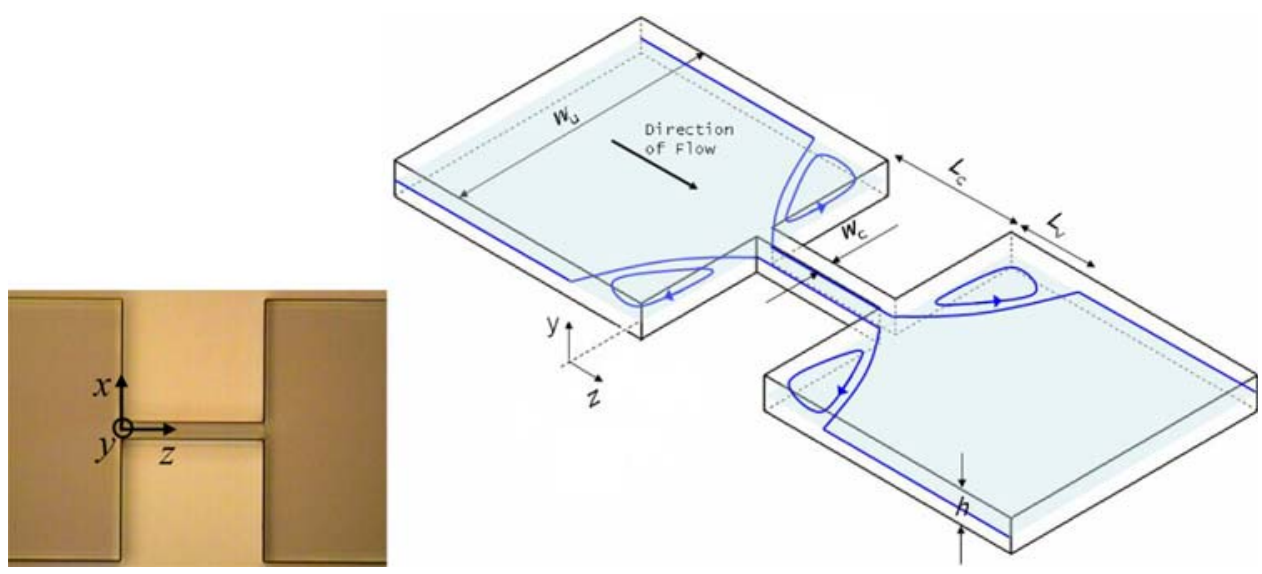
The $\mu \mathrm{PIV}$ technique uses the basic PIV principle of measuring the local velocities from the average displacement of tracer particles in a correlation region over a known time (Wereley and Meinhart 2004). Fluorescent particles $(\mathrm{Ex} / \mathrm{Em}=520 / 580 \mathrm{~nm})$ with $0.5 \mu \mathrm{m}$ diameter were added to the fluid at a concentration of $0.05 \%$ and a double-pulsed $532 \mathrm{~nm}$ Solo Nd:YAG laser system (New Wave Research) was used to illuminate the flow. A digital CCD camera $(1,375 \times 1,040$ pixels $)$ connected to the Nikon microscope with a $20 \mathrm{X}$ objective lens $(\mathrm{NA}=0.5)$ was used to capture the resulting images of particle displacement. In this configuration, the measurement depth is $11.7 \mu \mathrm{m}$ (Oliveira et al. 2007a), which corresponds to $21 \%$ of the channel depth. In order to optimally resolve the velocities in the section to be analyzed, the time separation $(\delta t)$ between individual images was varied depending on the flow rate used, so that the particle displacements (along the centerline) were within the range $1.5 d_{\mathrm{p}} \leq \delta z \leq 8 d_{\mathrm{p}}$, where $d_{\mathrm{p}}$ is the particle diameter and $\delta z$ is the particle displacement. For each experiment, a set of 25 image pairs were recorded, processed and ensemble averaged. Each image was crosscorrelated in interrogation areas of $32 \times 32$ pixels using a Nyquist algorithm with a $50 \%$ overlap to generate 2D velocity vector maps. A detailed discussion on the resolution and accuracy of the $\mu \mathrm{PIV}$ technique is presented in Oliveira et al. (2007a).

The pressure drop was measured experimentally across a section of the channel containing the contractionexpansion, using solid-state differential pressure sensors connected via flexible tubing to two pressure taps located $3 \mathrm{~mm}$ upstream and downstream of the contraction plane.

\section{Numerical simulations}

\subsection{Governing equations and numerical method}

The governing equations for an isothermal incompressible fluid flow are the conservation of mass and momentum, expressed as follows:

$\nabla \cdot \mathbf{u}=0$

$\rho\left[\frac{\partial \mathbf{u}}{\partial t}+(\mathbf{u} \cdot \nabla) \mathbf{u}\right]=-\nabla p+\nabla \cdot \tau$

where $\rho$ is the density of the fluid, $t$ the time, $\mathbf{u}$ the velocity vector, $p$ the pressure and $\tau$ the extra stress tensor. An appropriate constitutive equation is needed for $\tau$. Since in this work we are interested in Newtonian fluid flows, then

$\tau=\mu\left(\nabla \mathbf{u}+\nabla \mathbf{u}^{\mathrm{T}}\right)$

where $\mu$ is the constant shear viscosity of the fluid.
In Newtonian calculations Eq. (3) is usually substituted in Eq. (2) and the well-known Navier-Stokes equations are then obtained. However, the numerical code used in this work has been developed primarily for differential viscoelastic models (of which Eq. (3) is a particularly simple limiting explicit case) and as such we keep Eq. (3) separated from Eq. (2), as in previous works (e.g. Oliveira et al. 1998; Oliveira and Pinho 1999). For all practical purposes this separation makes no difference, except for the additional memory used to allocate storage for the extra stress tensor. In the work of Oliveira and Pinho (1999), a comparison between the formulation used in this work and the classical approach is presented.

Equations (1)-(3) assume the validity of the continuum hypothesis, an assumption which has been questioned in a number of works related to microfluidic applications (Pit et al. 2000; Barrat and Bocquet 1999). Some preliminary experiments involving liquids in lyophobic channels have observed slip occurring at the solid boundary (cf. the review by Neto et al. 2005). However, as far as current techniques permit, for Newtonian liquid flows at the micrometer length scales it has been well established that under standard conditions the basic laws governing fluid flow, expressed by the conservation of mass and momentum equations and the no-slip boundary condition at the walls, remain valid (Whitesides and Stroock 2001; Rodd et al. 2005; Karniadakis et al. 2005). For water, the continuum assumption is not expected to break down when the channel dimensions are above $1 \mu \mathrm{m}$ (Gad-el-Hak 2002). In fact, a $10 \mu \mathrm{m}$ thickness channel contains about $3 \times 10^{4}$ water molecules (considering a diameter of $\approx 0.3 \mathrm{~nm}$ for a water molecule), enough to treat the flow under the continuum theory (Bayraktar and Pidugu 2006). The agreement between experimental results and the numerical simulations here presented provides further credibility to this assumption.

A finite-volume method is used to solve Eqs. (1)-(3) numerically, using a time marching algorithm (Oliveira et al. 1998; Alves et al. 2000). In this methodology, the resulting algebraic equations relate the dependent variables $(p, \mathbf{u}, \tau)$, which are calculated at the center of the cells forming the computational mesh, to the values in the nearby surrounding cells. Orthogonal non-uniform blockstructured meshes are used to map the computational domain. A time-marching algorithm is used to approach the steady-state solution and an implicit first-order Euler scheme is used in the discretization of the time derivative. We note that because we are only concerned with steady flow conditions in this work, the precision in the time derivative is irrelevant since, under steady conditions, the time derivative term vanishes. Central differences are used to discretize the diffusive terms, while the CUBISTA highresolution scheme (Alves et al. 2003) is used in the 
discretization of the advective terms of the momentum equations. This scheme is based on the QUICK scheme of Leonard (1979) and maintains its third-order accuracy while ensuring boundedness and good iterative-convergence properties (Alves et al. 2003, 2004).

The physical properties of the fluid used in the numerical calculations were selected to match those measured experimentally, i.e. the viscosity was set to $0.94 \mathrm{mPa}$ s and the density to $998 \mathrm{~kg} / \mathrm{m}^{3}$.

\subsection{Problem definition and dimensionless numbers}

In order to be able to capture the flow asymmetries expected at large $R e$ the full domain is used in the simulations, i.e. no symmetry boundary conditions are imposed. The geometry used for the computations is the same as in the experiments, as illustrated in Fig. 1. The dimensions of the inlet and outlet lengths were set to be longer $\left(L_{\mathrm{u}}=L_{\mathrm{d}}=30 w_{\mathrm{u}}\right)$ than the experimental device to ensure that the flow fully develops upstream of the contraction and completely re-develops downstream of the expansion. This is also required in order to obtain precise values of the excess pressure drop due to the presence of the contraction-expansion. Three different contraction lengths were simulated to match experiments: $L_{\mathrm{c}}=100,200$ and $400 \mu \mathrm{m}$. As mentioned in the Introduction, there are two important geometrical ratios to be considered in contraction-expansion flows: the aspect ratio and the contraction ratio (or expansion ratio). The contraction ratio is defined as $\mathrm{CR}=w_{\mathrm{u}} / w_{\mathrm{c}}$ and was kept constant throughout this study to match the experimental conditions described in Sect. 2.1. The aspect ratio is defined as $\mathrm{AR}=h / w_{\mathrm{c}}$. In the experimental study, a single channel depth of $h=55 \mu \mathrm{m}$ was used, while in the numerical simulations, the depth of the channel was varied from 0.1 to $800 \mu \mathrm{m}$, i.e. the aspect ratio was varied by almost four orders of magnitude, in order to analyze its influence on the resulting flow kinematics and on the pressure drop. The limiting case of $2 \mathrm{D}$ flow $(\mathrm{AR} \rightarrow \infty)$ is also analyzed in detail.

The other important dimensionless variable that characterizes the flow behavior of an incompressible Newtonian fluid is the Reynolds number $(R e)$ as shown in Fig. 2. Control volume analysis of fully developed flow leads to the concept of a hydraulic diameter described in terms of the cross-sectional area and wetted perimeter of the channel cross-section: $D_{\mathrm{h}}=4$ Area/Perimeter (Gad-el-Hak 2002). This quantity provides a simple way of characterizing a channel of non-circular cross-section using a single characteristic length scale. In microfluidics, where we are commonly dealing with channels of planar cross-section, it is typical to define $R e$ as a function of the hydraulic diameter of the contraction channel, $D_{\mathrm{h}, \mathrm{c}}=2 h w_{\mathrm{c}} /\left(h+w_{\mathrm{c}}\right)$,
Fig. 2 a Effect of mesh refinement on the normalized axial velocity $\left(V_{z} /\left\langle V_{z}\right\rangle_{\mathbf{u}}\right)$ contour lines at the center plane $(y=0)$ upstream and downstream of the contraction obtained numerically with the coarse mesh (dashed lines) and the refined mesh (solid lines) for the $3 \mathrm{D}$ geometry $\left(w_{\mathrm{c}}=26 \mu \mathrm{m}\right.$, $\left.h=55 \mu \mathrm{m}, L_{\mathrm{c}}=100 \mu \mathrm{m}\right)$. b Zoomed view of the refined mesh near the contractionexpansion region

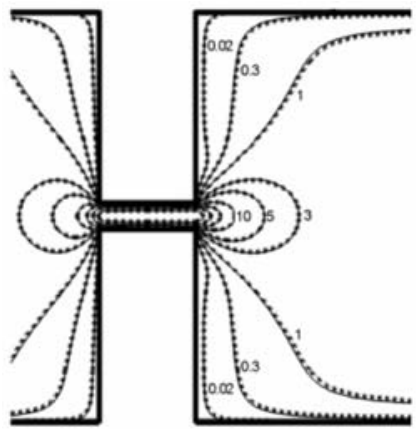

$\operatorname{Re}=7.3$

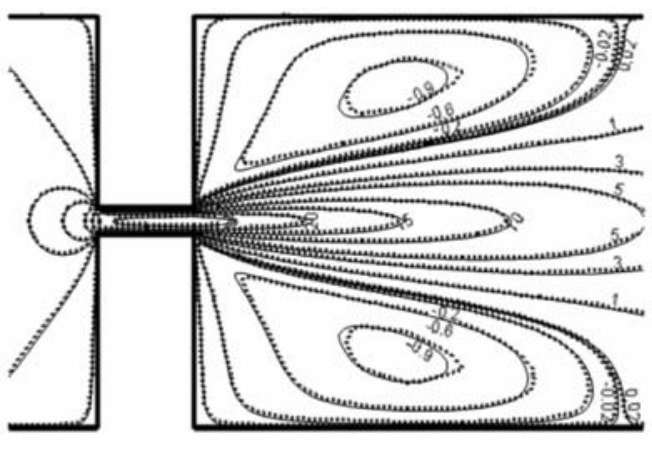

$\operatorname{Re}=73$

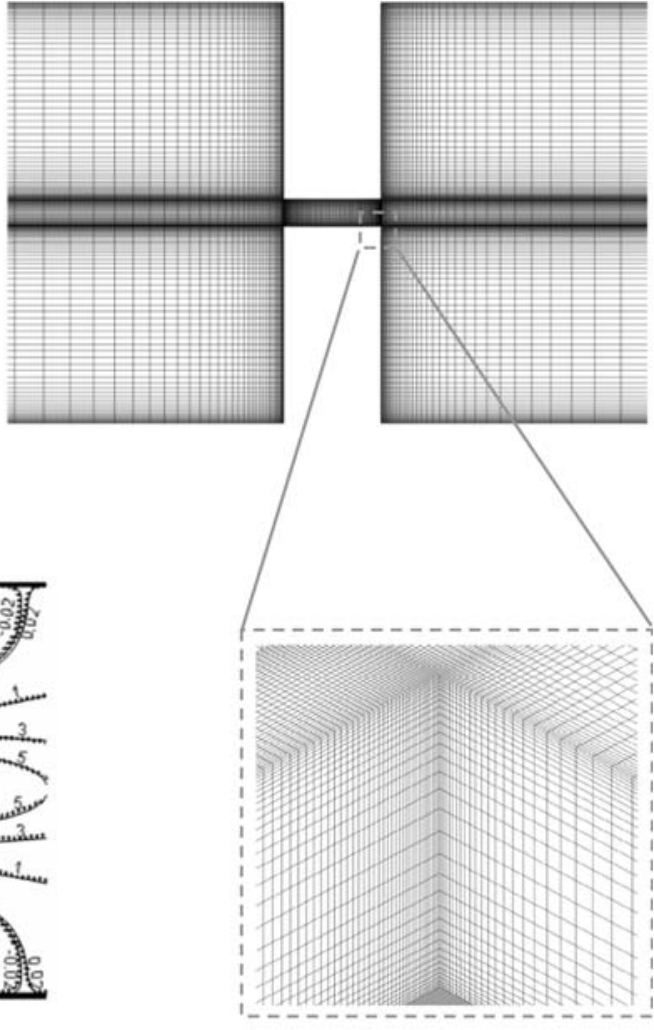

(b) 
and the average velocity in the contraction region $\left(\left\langle V_{z}\right\rangle_{\mathrm{c}}=Q / h w_{\mathrm{c}}\right.$, where $Q$ is the volumetric flow rate) (Rodd et al. 2005; Tsai et al. 2006) resulting in:

$R e=\frac{\rho\left\langle V_{z}\right\rangle_{c} D_{\mathrm{h}, \mathrm{c}}}{\mu}=\frac{\rho\left\langle V_{z}\right\rangle_{\mathrm{c}} w_{\mathrm{c}}}{\mu} \frac{2 \mathrm{AR}}{\mathrm{AR}+1}$

For 2D flows, in which $h / w_{\mathrm{c}}>>1, D_{\mathrm{h}, \mathrm{c}} \rightarrow 2 w_{\mathrm{c}}$ and the Reynolds number becomes $\operatorname{Re}_{(2 \mathrm{D})}=\frac{2 \rho\left\langle V_{z}\right\rangle_{\mathrm{c}} w_{\mathrm{c}}}{\mu}$. Alternatively, the Reynolds number can be defined directly in terms of the contraction width, $R e_{\mathrm{w}_{\mathrm{c}}}=\frac{\rho\left\langle V_{z}\right\rangle_{\mathrm{c}} w_{\mathrm{c}}}{\mu}$, i.e. $R e_{\mathrm{w}_{\mathrm{c}}}=$ $R e \frac{\mathrm{AR}+1}{2 \mathrm{AR}}$, which in some cases can be a more convenient representation of the flow behavior, as will be discussed in Sect. 6.

Computational meshes with different levels of refinement were used to map each contraction-expansion geometry. The meshes are orthogonal but non-uniform with the size of each cell relating to its neighbors by a geometric progression within each direction. The smallest cells are concentrated near the re-entrant corners, where stress and velocity gradients are expected to be higher. For the 3D geometries, the size of the smallest cell normalized by the width of the contraction is $\Delta x_{\min } / w_{\mathrm{c}}=\Delta y_{\min } / w_{\mathrm{c}}=$ $\Delta z_{\min } / w_{\mathrm{c}}=0.04$ for the coarser meshes and half this value for the refined meshes (cf. Fig. $2 b$ for an example of a refined mesh), which were obtained by doubling the number of cells in each direction. For the range of flow conditions studied, the results are mesh independent within $2 \%$ (cf. the axial velocity contour plots shown in Fig. 2a). Therefore, unless stated otherwise, the results presented in the remainder of the paper were obtained with the coarser mesh. In the limiting 2D case, where we study a much broader range of flow rates, the effect of mesh refinement can be more noticeable at higher flow rates. Also, to be able to capture accurately the critical conditions for onset of flow asymmetries and bifurcations, meshes with high spatial refinement are necessary. Therefore, the $2 \mathrm{D}$ results presented in this work were computed with meshes that are more refined in the $x-z$ plane. In the $2 \mathrm{D}$ case, $\Delta x_{\min } / w_{\mathrm{c}}=$ $\Delta z_{\min } / w_{\mathrm{c}}=0.02$ for the coarser meshes and $\Delta x_{\min } / w_{\mathrm{c}}=$ $\Delta z_{\min } / w_{\mathrm{c}}=0.01$ for the refined meshes.

\section{Flow patterns and recirculation characteristics}

\subsection{Experimental microfluidic device (3D computations)}

At low Reynolds numbers the diverging flow downstream of the expansion plane is symmetric about the midplane $(x=0)$ of the device as shown in Fig. 3. In this figure we overlay the experimental streak lines measured at the center plane $(y=0)$ downstream of the expansion plane with the corresponding numerical calculations. There is a good agreement between the experimental streak lines and the numerical predictions, which can be further confirmed in Fig. 4. An increase in $R e$ leads to the development and enhancement of recirculating "lip vortices" $(R e \lesssim 20)$, as well as reattached symmetric corner recirculations at higher Reynolds number, downstream of the expansion plane (Fig. 3). This is in agreement with the observations of Townsend and Walters (1994), for a 14:1 expansion geometry, and Rodd et al. (2005), for a similar geometry to the one used here. In Fig. 4, we illustrate the effect of inertia on the size of the primary recirculations formed downstream of the expansion as the Reynolds number is increased. The dimensionless size of the lip recirculation that is formed at low $R e$ is defined by its length in the $x$-direction normalized with $w_{\mathrm{u}}\left(L_{\mathrm{lip}} / w_{\mathrm{u}}\right.$, see Fig. 3$)$ and is limited to a maximum of $\left(w_{\mathrm{u}}-w_{\mathrm{c}}\right) /\left(2 w_{\mathrm{u}}\right)=0.47$. The strength and size of the recirculations increase as the effects of inertia increase and once they reach the salient corner, the recirculations continue to grow in the downstream direction. Their size $\left(L_{\mathrm{V}}\right)$ is now parameterized by the recirculation length in the $z$-direction as illustrated in Fig. 3 (and by convention they are now termed corner recirculations or "corner vortices"). It should also be noted that the streak lines and recirculation sizes measured experimentally are symmetric about the centerline even for the highest $R e$ tested.

At first glance, the experimental streak lines and the numerical predictions in Fig. 3 may suggest that the recirculations are closed structures. In fact, in a 3D geometry, the presence of a top and bottom bounding wall leads to complex 3D spiraling recirculation structures (Chiang et al. 2000; Tsai et al. 2006). The fluid enters the recirculating structure close to the wall and follows a spiral movement toward the center plane, where it exits the recirculation. Nevertheless, despite the different circulation patterns and the fact that the flow is highly three-dimensional, the sizes of the projected recirculations near the wall and at the center plane are very similar. Thus, even though the experimental streakline images arise from fluorescent particles over approximately 54\% of the channel depth (as explained in Sect. 2.2), they are visually very clear and sharp.

The complex path of two fluid elements in a 3D geometry is illustrated in Fig. 5, where the open nature of the recirculation is clearly evident. To obtain the streak lines displayed, the two fluid elements were selected in order to pass a point downstream of the contraction with the same axial and lateral coordinates, $z=500 \mu \mathrm{m}$ and $x=180 \mu \mathrm{m}$, but with different out-of-plane coordinates: $y=1 \mu \mathrm{m}(1 \mu \mathrm{m}$ from the center plane; fluid element 1$)$ and $y=21.5 \mu \mathrm{m}$ (6 $\mu \mathrm{m}$ from the wall; fluid element 2$)$. In this way, we capture one of the particles that is entrained within the recirculation and another one that just manages 
Fig. 3 Effect of inertia on the experimental streaklines (photograph) and predicted streamlines (solid lines) obtained at the center plane $(y=0)$ downstream of the contraction for water flowing through a 400:26:400 contraction-expansion with $h=55 \mu \mathrm{m}$ and $L_{\mathrm{c}}=200 \mu \mathrm{m}$

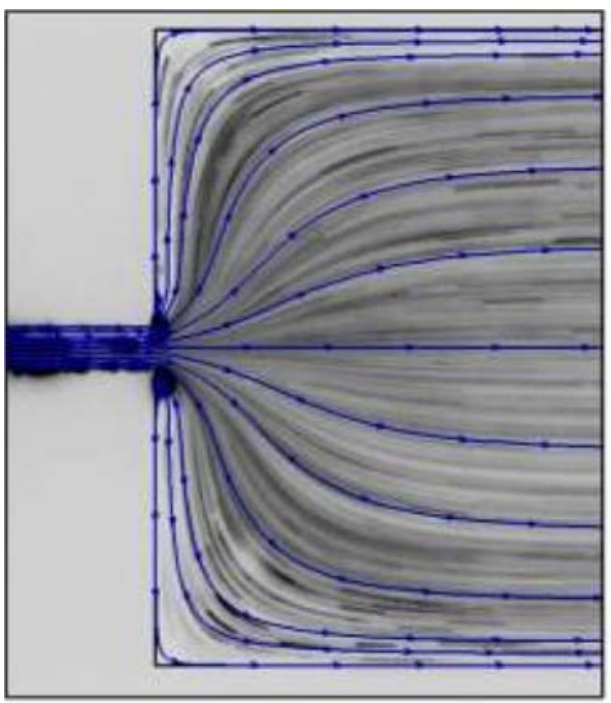

$R e=7.3$

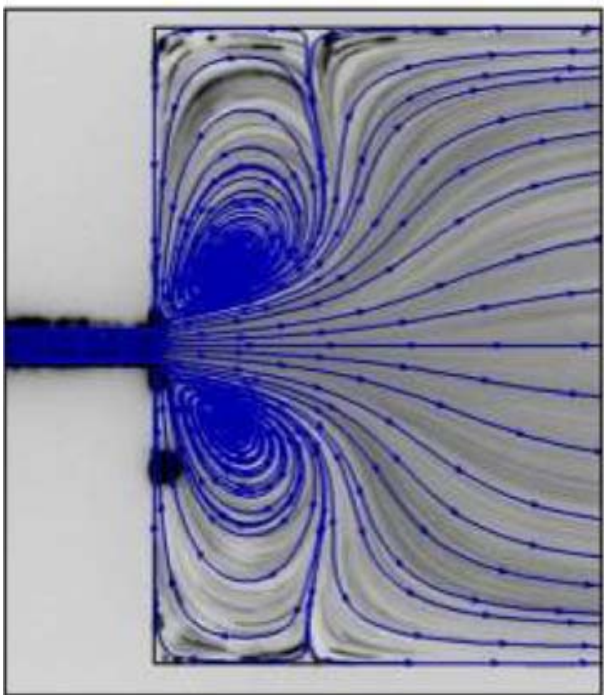

$\operatorname{Re}=25.5$

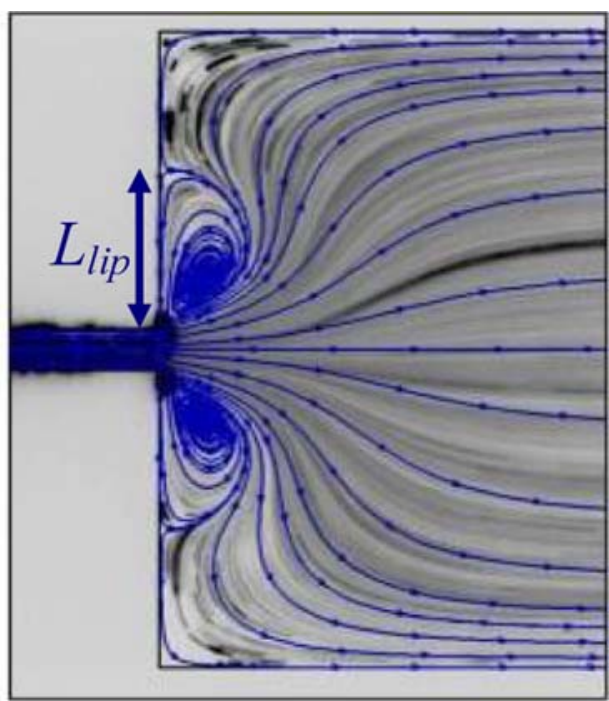

$\operatorname{Re}=18.2$

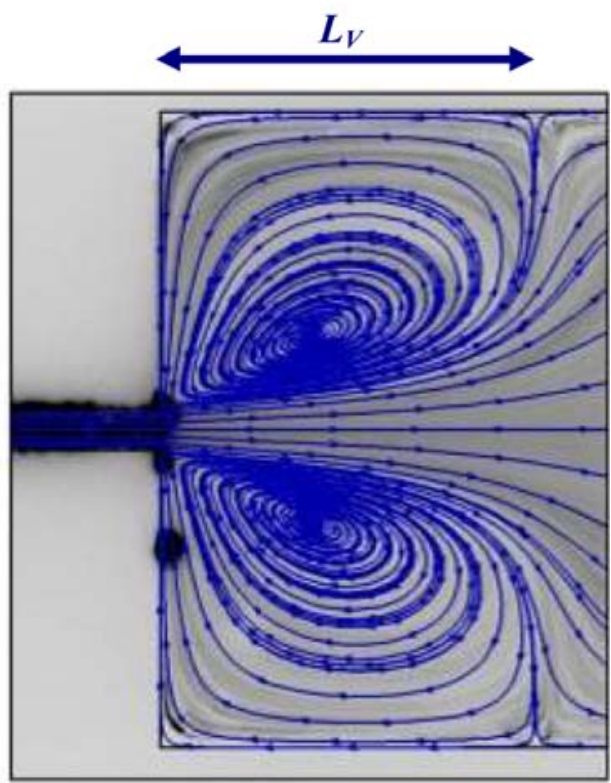

$\operatorname{Re}=43.7$ to avoid it. An analysis of the Lagrangian trajectories of the two particles shows a large difference in residence times. Even though fluid element 2 spends most of the time close to the upper bounding wall, where the velocities are lower, its residence time is smaller since it is not trapped in the recirculation (see Supplementary Information, Sect. S1).

\subsection{Effect of the aspect ratio}

In such a markedly three-dimensional flow, the channel depth is bound to have an impact on the flow patterns. Figure 6 illustrates the effect of varying the aspect ratio AR on the predicted streamlines. The numerical calculations were obtained for $L_{\mathrm{c}}=200 \mu \mathrm{m}$, keeping the Reynolds number constant $(R e=43.7$, as defined in Eq. (4)) while varying the channel depth $h$. The primary recirculations are seen to expand as the channel depth is increased, while keeping the Reynolds number constant. It is interesting to note that, for this chosen value of $R e$, the flow becomes asymmetric at an aspect ratio (AR $\left.=h / w_{\mathrm{c}}\right)$ between $100 / 26$ and 400/26. Thus, by reducing the strength of secondary flows and inhibiting the onset of flow asymmetries, the walls have a stabilizing effect on the flow patterns. It is important to notice that we only observe asymmetries at rather high aspect ratios for the range of Reynolds numbers analyzed. We re-emphasize that this transition represents a bifurcation to an asymmetric flow structure that is steady in time. In fact, all the results presented in this work (both computational and experimental) are for steady-state flow conditions. For the 3D geometry used in the experiments 


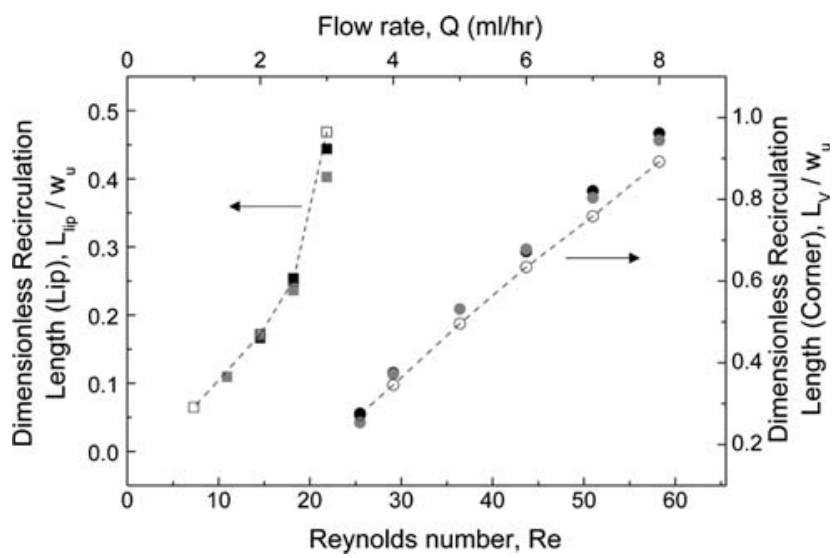

Fig. 4 Effect of inertia on the dimensionless recirculation length at the center plane $(y=0)$ for the experimental geometry $\left(w_{\mathrm{c}}=26 \mu \mathrm{m}\right.$, $\left.h=55 \mu \mathrm{m}, L_{\mathrm{c}}=200 \mu \mathrm{m}\right)$. The open symbols represent the numerical data; the dark and light filled symbols represent the experimental measurements of the upper and lower recirculation sizes, respectively

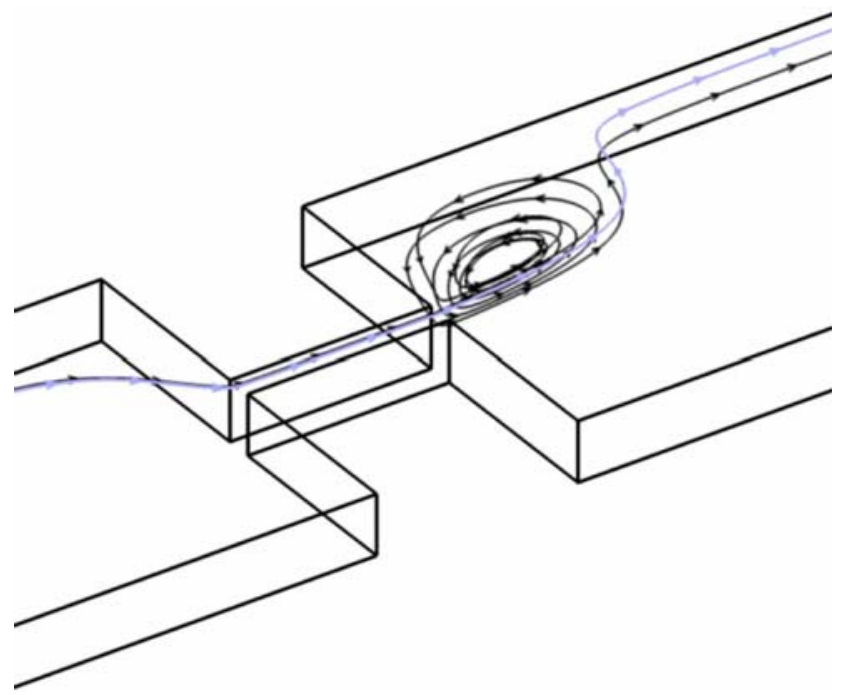

Fig. 5 Trajectories of two material points through the sudden contraction-expansion $\left(w_{\mathrm{c}}=26 \mu \mathrm{m} ; L_{\mathrm{c}}=200 \mu \mathrm{m} ; \quad h=55 \mu \mathrm{m}\right)$, one that enters the recirculation (dark line) and goes through point $z=500 \mu \mathrm{m}, x=180 \mu \mathrm{m}$ and $y=1 \mu \mathrm{m}$ and another that does not enter the recirculation (light line) and goes through point $z=500 \mu \mathrm{m}, x=180 \mu \mathrm{m}$ and $y=21.5 \mu \mathrm{m}$

$\left(\mathrm{AR}=h / w_{\mathrm{c}}=2.12\right)$ no flow asymmetries were observed even for a Reynolds number as high as $R e=110$.

With the objective of performing a systematic study of the effect of the aspect ratio on the flow structure, we have carried out an extensive set of simulations and classified the patterns of the flow downstream of the contraction at increasing Reynolds number. These calculations were performed for $L_{\mathrm{c}}=400 \mu \mathrm{m}$ and the resulting flow structures were classified as "no recirculation", "lip recirculation", "corner recirculation", "combination of lip and corner recirculations", "full corner recirculation" and "asymmetric flow structure". The flow pattern map in Fig. 7 illustrates these flow structures and their location in the $\mathcal{H}-\operatorname{Re}$ parameter space. We have chosen the dimensionless variable $\mathcal{H} \equiv h /\left(h+w_{\mathrm{c}}\right)=\mathrm{AR} /(\mathrm{AR}+1)$ as the appropriate geometric ratio to plot, instead of the raw aspect ratio AR defined previously, so the values of the ordinate axes are bounded between zero (AR $\rightarrow 0$; corresponding to the Hele-Shaw flow limit) and unity (AR $\rightarrow \infty$; corresponding to the 2D flow limit).

In the lower left corner of Fig. 7, which represents low $\mathcal{H}$ and low $R e$, there is no visible recirculation formed downstream of the expansion and the flow patterns resemble that of irrotational flow. On the other hand, in the opposite corner of high $\mathcal{H}$ and high $R e$, asymmetric flow with large recirculating structures, is observed. In-between these two regions, we find a combination of lip and/or corner recirculations. Independent of the spatial structure of the flow, recirculation enhancement with increasing fluid inertia is seen for all aspect ratios considered, except in the limit of $\mathcal{H} \rightarrow 0$, for which we have a Hele-Shaw flow, and therefore the flow in the $z-x$ plane is irrotational without any recirculation. The formation of a small and weak recirculation near the corner (the "Moffatt eddy"), appears to occur at a fixed value of $\mathcal{H} \approx 0.5$ (corresponding to $\mathrm{AR} \approx 1$ ) independently of the Reynolds number. Increasing the Reynolds number leads to the onset of a lip recirculation, near the re-entrant corner, which increases in size and intensity until it reaches the downstream wall, creating a large corner recirculation. Further increases in $R e$ leads to a bifurcation to an asymmetric flow at high $\mathcal{H}$ as already discussed.

\subsection{Two-dimensional geometry (2D)}

As noted in the Introduction, the fluid motion approaches a truly 2D flow when AR $>1$ (Chiang et al. 2000; Tsai et al. 2006), and is prone to develop asymmetries in the downstream channel as the Reynolds number increases (e.g. Oliveira 2003). In Fig. 8, we examine the onset of these asymmetries in more detail by showing the streamlines calculated numerically for $2 \mathrm{D}$ flow as a function of $R e$. The Reynolds number in the 2D geometry is given by $\operatorname{Re}_{(2 \mathrm{D})}=\rho\left\langle V_{z}\right\rangle_{\mathrm{c}} 2 w_{\mathrm{c}} / \mu$, as already explained in Sect. 3.2. It should be noted that many authors studying 2D flows in expansion geometries prefer to use $R e=\rho\left\langle V_{z}\right\rangle_{\mathrm{c}} w_{\mathrm{c}} / \mu$, which is half of the value reported here. For low Reynolds number (e.g. $R e_{(2 \mathrm{D})}=0.01$ ), the viscous flow through the contraction-expansion geometry is reversible (fore/aft symmetric) as shown in Fig. 8a and it is impossible to deduce the flow direction. For $R e_{(2 \mathrm{D})}=1.3$ (Fig. 8b), the small Moffatt eddy upstream of the contraction reduces in size slightly, while the vortex downstream of the expansion 
Fig. 6 Effect of channel depth on the predicted streamlines at the center plane $(y=0)$ for a 400:26:400 contractionexpansion geometry with $L_{\mathrm{c}}=200 \mu \mathrm{m}$, keeping the Reynolds number constant $(R e=43.7)$

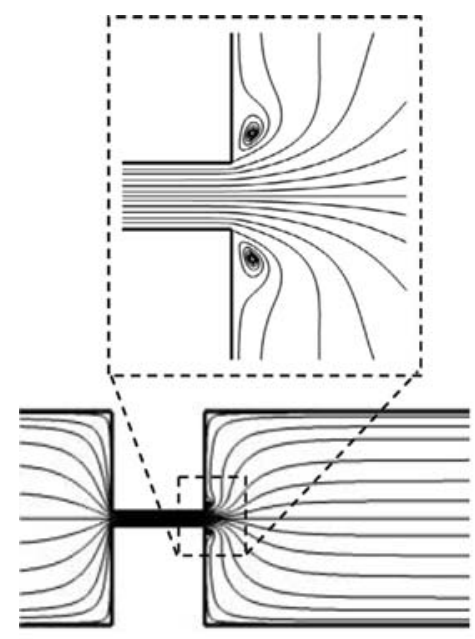

$h=40 \mu \mathrm{m}(\mathrm{AR}=1.54)$

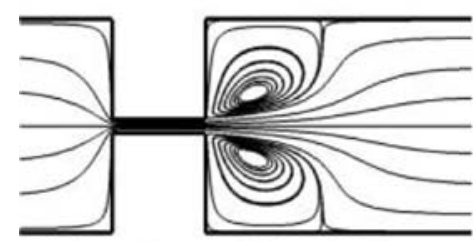

$h=55 \mu \mathrm{m}(\mathrm{AR}=2.12)$

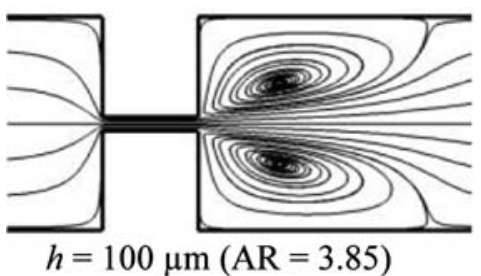

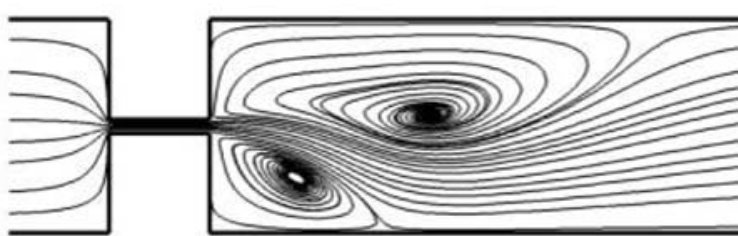

$h=400 \mu \mathrm{m}(\mathrm{AR}=15.4)$

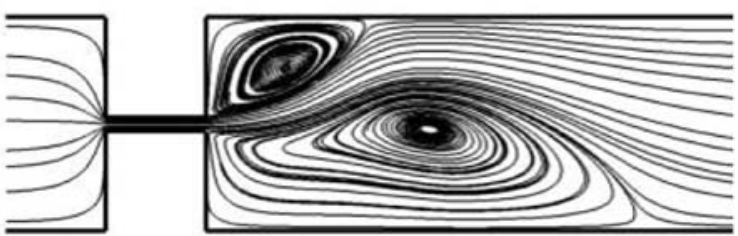

$h=800 \mu \mathrm{m}(\mathrm{AR}=30.8)$

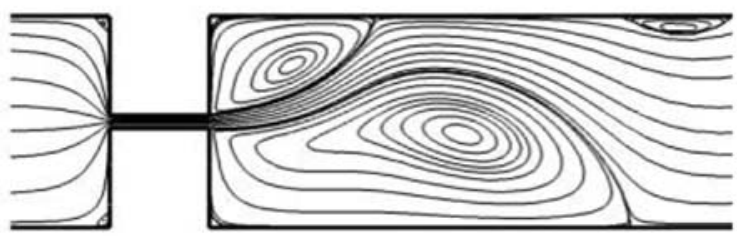

2D

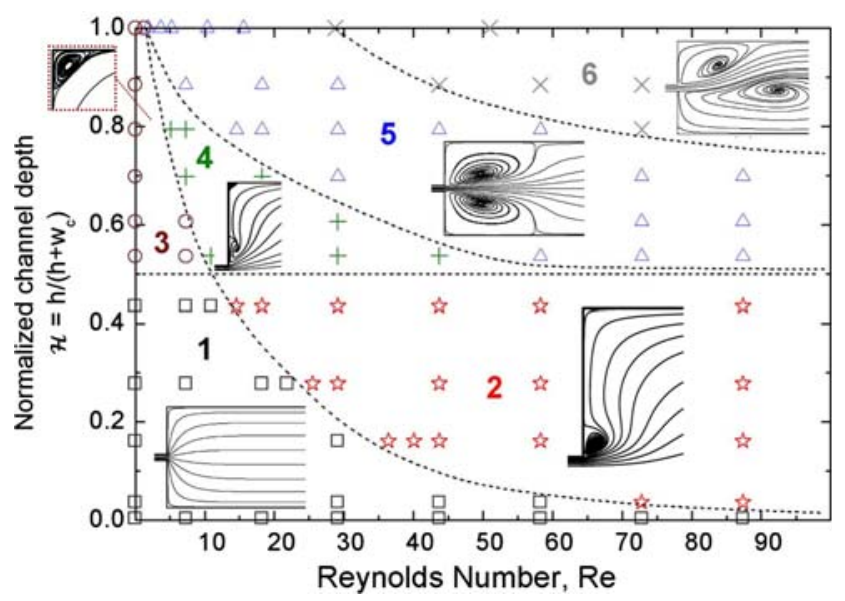

Fig. 7 Flow pattern map: 1 no recirculation; 2 lip recirculation; 3 corner recirculation; 4 lip and corner recirculations; 5 full corner recirculation; 6 asymmetric flow

plane increases in size and intensity. It is clear that in the limiting case of a 2D geometry, the vortices are now closed recirculating structures. The flow remains symmetrical about the center plane $(x=0)$ as the Reynolds number is further increased and, in addition, the corner vortices downstream of the expansion grow while the upstream corner vortices get smaller, as indicated by the streamline plots in Fig. 8b-c. On further increasing $R e_{(2 \mathrm{D})}$, we find that the 2D flow exhibits a supercritical bifurcation becoming asymmetric with respect to the $x=0$ line (Fig. 8d). We note once again that the pattern presented in Fig. 8d was obtained under steady-state conditions and could have evolved to this or its image configuration with respect to the $x=0$ line. At $R e_{(2 \mathrm{D})}=71.3$ (Fig. 8e), a third large vortex has already emerged downstream of the expansion.

The evolution of the vortex structures can be summarized in the bifurcation diagram of Fig. 9, in which we show the effect of the Reynolds number on the size of the vortices formed downstream of the 2D planar expansion. The lengths corresponding to each branch of this bifurcation diagram (L1 to L4) are marked in Fig. 8e. The critical Reynolds number for the onset of asymmetric flow is approximately $\operatorname{Re}_{(2 \mathrm{D}) \mathrm{c}} \approx 28.5$. At $\operatorname{Re}_{(2 \mathrm{D})}=31.1$ the flow is 
Fig. 8 Predicted streamlines for a $2 \mathrm{D}$ contraction-expansion $(\mathcal{H}=1)$, with $w_{\mathrm{c}}=26 \mu \mathrm{m}$ and $L_{\mathrm{c}}=200 \mu \mathrm{m}$, over a range of Reynolds numbers: $0.01 \leq \operatorname{Re}_{(2 \mathrm{D})} \leq 71.3$

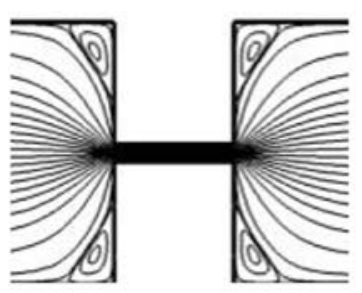

(a) $R e_{(2 \mathrm{D})}=0.01$

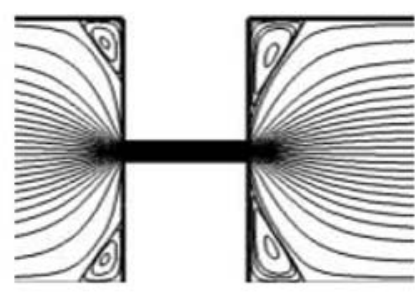

(b) $R e_{(2 \mathrm{D})}=1.3$

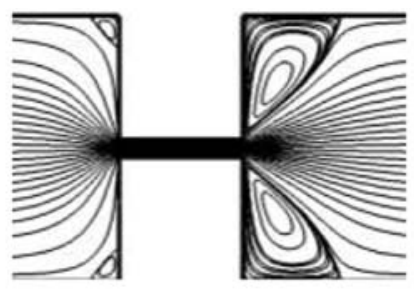

(c) $\operatorname{Re}_{(2 \mathrm{D})}=7.8$

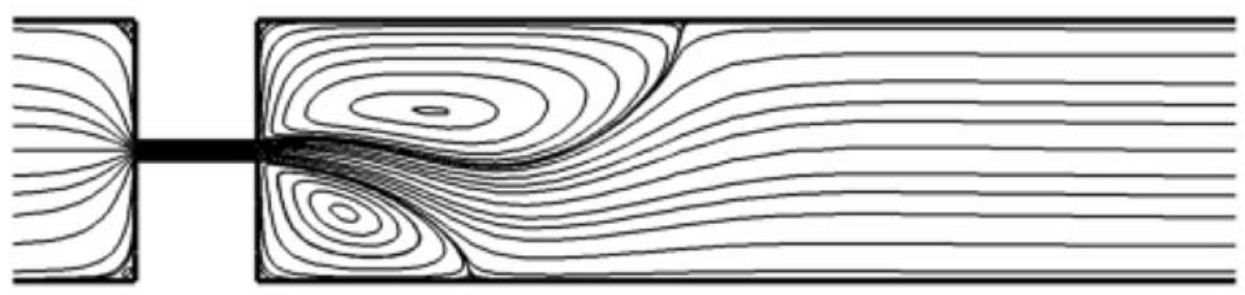

(d) $R e_{(2 \mathrm{D})}=31.1$

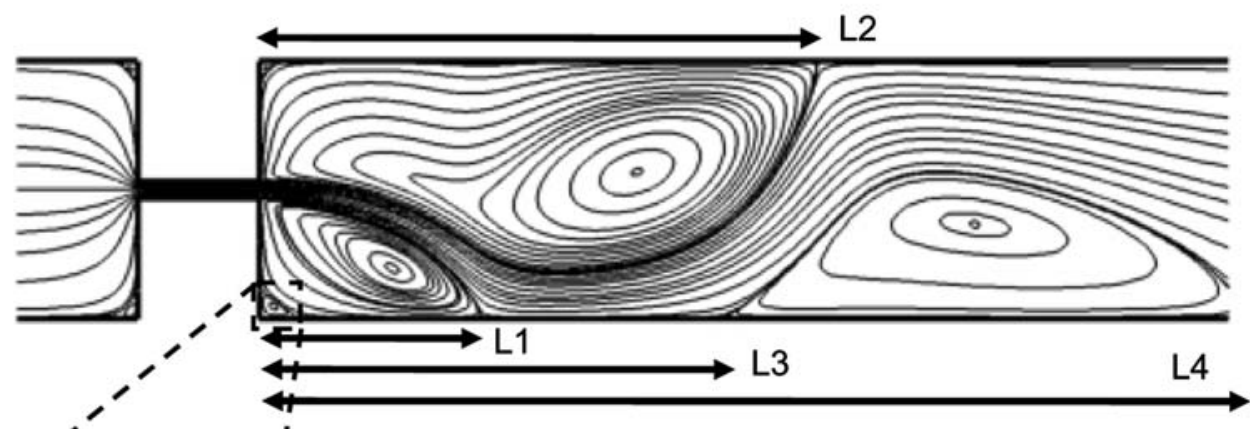

(e) $R e_{(2 \mathrm{D})}=71.3$ clearly asymmetric (Fig. 8d), but for $R e_{(2 \mathrm{D})}=28.5$, although not yet clearly visible in the bifurcation plot, a slight asymmetry is already present. This value is in good agreement with the computed results of Mishra and Jayaraman (2002) in a 1:16 2D expansion $\left(\operatorname{Re}_{(2 \mathrm{D}) \mathrm{c}}=25.2\right)$, even though the two works are not exactly comparable (here the contraction/expansion ratio is $\mathrm{CR}=15.4$ and the flow in the smaller channel may not be fully developed when the expansion is approached). Near the critical point, the larger recirculation increases in size while the smaller one decreases as the Reynolds number increases. For $R e_{(2 \mathrm{D})} \approx 42$, a new vortex forms downstream of the two primary vortices (Fig. 8e) on the side of the smaller corner vortex. In all cases discussed, for $\mathcal{H}=1$ (2D flow), we are able to identify smaller corner (Moffatt) vortices downstream of the expansion plane in addition to the primary vortices, as shown by the inset in Fig. 8e.

\section{Velocity field in the contraction-expansion}

The velocity field was determined experimentally using $\mu \mathrm{PIV}$ and compared to the numerical calculations. To assess the evolution of the velocity along the lateral direction as the contraction is approached, we have plotted both experimental and numerical velocity profiles for the center plane $(y=0)$ and for various axial positions upstream of the contraction $\left(-1.49 \leq z /\left(w_{\mathrm{u}} / 2\right) \leq 0.235\right)$. In Fig. 10a, we depict the transverse profiles of the axial velocity $V_{z}$ and in Fig. $10 \mathrm{~b}$ of the lateral velocity $V_{x}$, both normalized with the average axial velocity upstream of the contraction. For an axial position well upstream the contraction plane $\left(z /\left(w_{\mathrm{u}} / 2\right)=-1.49\right)$, the profiles approach those of fully developed flow in a channel of rectangular cross-section. As we move toward the contraction plane, the streamlines converge toward the centerline. This causes 


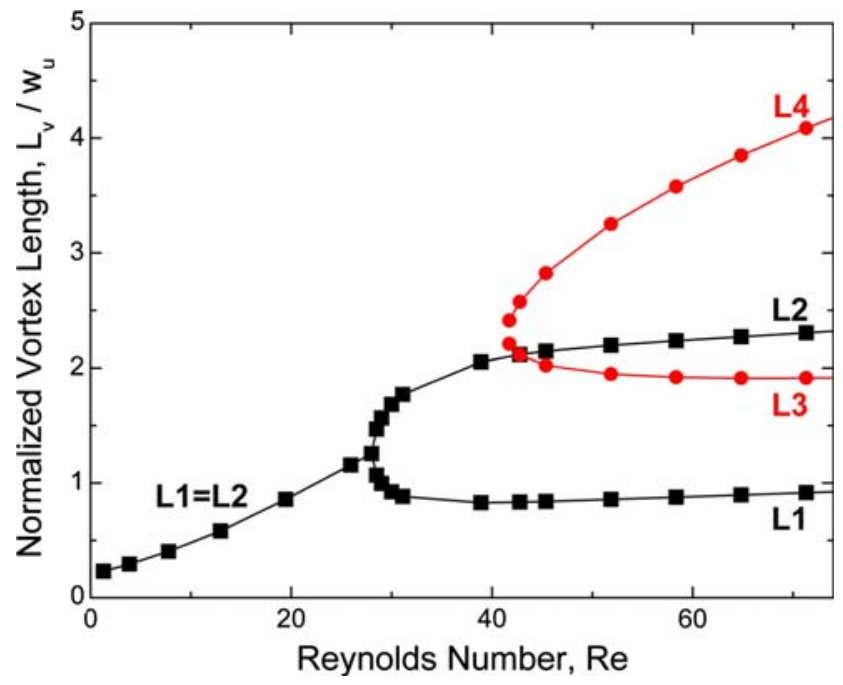

Fig. 9 Bifurcation diagram for a $2 \mathrm{D}$ geometry $(\mathcal{H}=1)$ with $w_{\mathrm{c}}=26 \mu \mathrm{m}$ and $L_{\mathrm{c}}=200 \mu \mathrm{m}$ : effect of Reynolds number, $\operatorname{Re}_{(2 \mathrm{D})}$

the axial velocity near the walls $\left(0.4 \lesssim\left|x /\left(w_{\mathrm{u}} / 2\right)\right| \lesssim 1\right)$ to decrease, while the lateral velocity increases substantially relative to the fully developed flow. The maximum velocity attained at the centerline $(y=0, x=0)$ increases as we approach $z=0$ and the effects of the contraction become more noticeable.

The experimental and predicted axial velocity profiles along the centerline upstream of the contraction are shown in Fig. 11 for $R e=7.3$. The axial velocity is scaled with the average upstream velocity and is compared to the equivalent numerical calculations. The profile evolves from fully developed in the upstream part of the channel far away from the contraction (where the centerline velocity is constant) to a region $\left(-5 \lesssim z / w_{\mathrm{c}} \lesssim 0\right)$ where the fluid accelerates as the contraction plane is approached. The numerical simulation is able to capture with good accuracy both the point at which the velocity starts to increase in the experiment, as well as the rate of increase (see also Supplementary Information, Sect. S2).

In Fig. 12a, we extend Fig. 11 (for $A R=2.12$ ) to show the computed axial velocity profiles over a larger range of axial positions along the centerline (including the upstream channel, the contraction and the downstream expansion) and for a broader range of $\operatorname{Re}(0.73 \leq R e \leq 43.7)$. Figure $12 \mathrm{~b}$ shows the corresponding strain rate profile $\left(\partial V_{z} / \partial z\right)$ along the centerline. The expected symmetrical behavior of the velocity profile, characteristic of creeping flows, can be readily observed for the lower Reynolds numbers. For the lowest Reynolds number $(R e=0.73)$, the velocity and velocity gradient profiles are symmetric. Consequently, just by looking at streak patterns we would not be able to identify which direction the fluid is flowing, since at this low Reynolds number the flow is reversible and the streak lines upstream and downstream of the contraction are
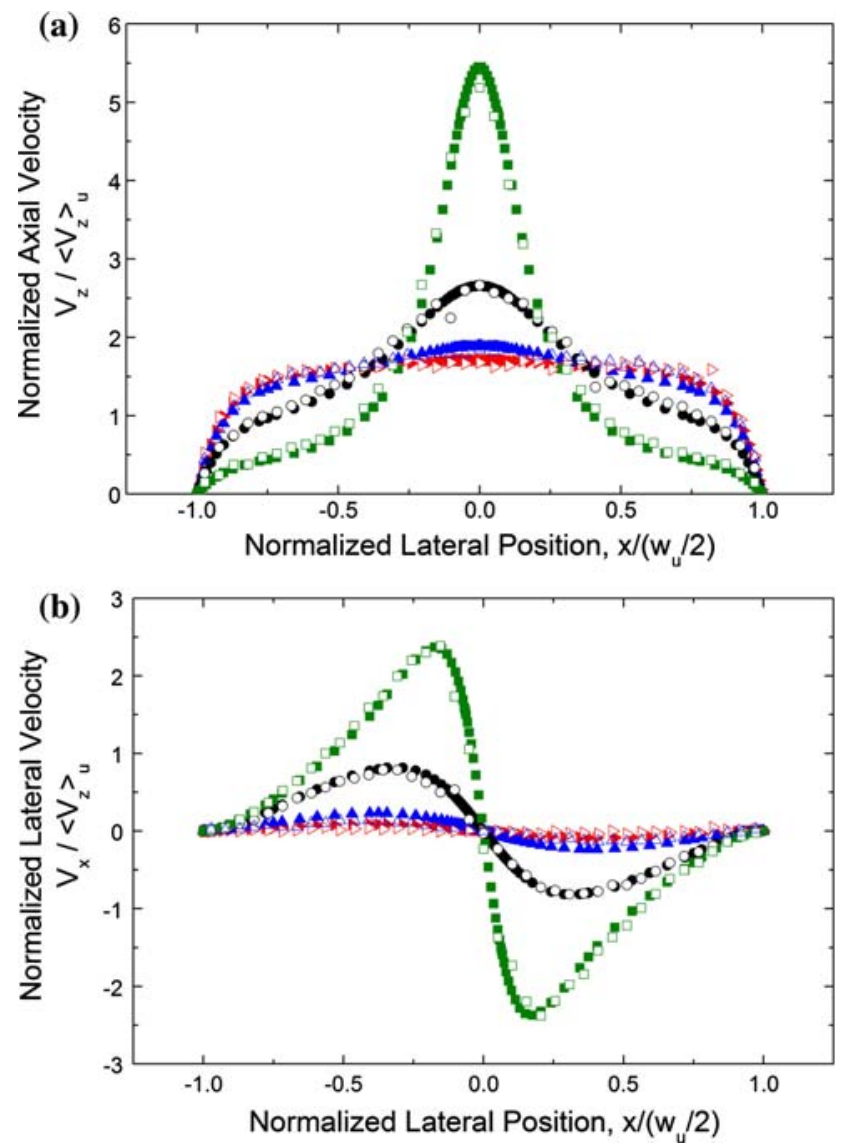

Fig. 10 Comparison of the dimensionless velocity profiles at the center plane $(y=0)$ for the contraction-expansion geometry ( $w_{\mathrm{c}}=26 \mu \mathrm{m}, h=55 \mu \mathrm{m}$ ) determined experimentally (open symbols) and numerically (filled symbols), as a function of the distance from the centerline for $R e=7.3$ and a range of $z$-positions upstream of the contraction plane denoted by the following dimensional and dimensionless positions: $z\left(z /\left(w_{\mathrm{u}} / 2\right)\right)$ : (square $)-47 \mu \mathrm{m}(-0.235)$, (circle) $-98 \mu \mathrm{m}$ (-0.49), (triangle) $-195 \mu \mathrm{m}$ (-0.975), (tilted triangle $)-298 \mu \mathrm{m}(-1.49)$. a Axial velocity; b lateral velocity

coincident. In this case, due to the small depth of the geometry $(\mathrm{AR}=2.12)$, the flow in the $z-x$ plane approaches a potential flow and is very similar to that observed in a Hele-Shaw apparatus. Also of interest is the absence of the Moffatt corner vortex (cf. region 1 in Fig. 7) characteristic of 2D viscous corner flows (Moffatt 1964). This vortex is present even under inertialess flow conditions in simulations of $2 \mathrm{D}$ flows and in general for high $\mathrm{AR}$ (AR $>>1$ ) (cf. region 3 in Fig. 7 and see Supplementary Information, Sect. S3).

For higher flow rates $(R e \geq 1)$, the profiles shown in Fig. 12 are no longer symmetric about the line $z=L_{\mathrm{c}} / 2$, and it takes longer for the velocity to decay toward its fully developed state. In addition, for most of these flow rates, the flow does not have a long enough residence time to fully develop in the contraction throat (see inset in Fig. 12a). Therefore, the results downstream of the 


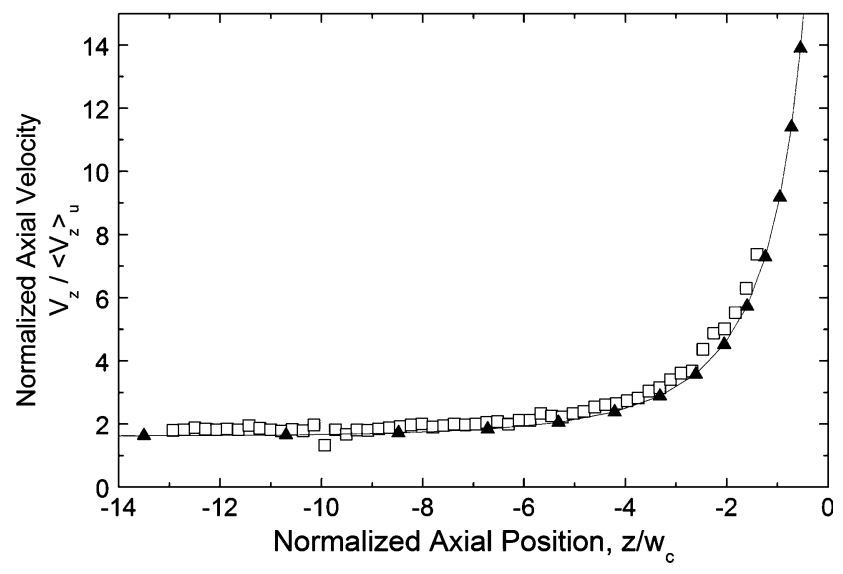

Fig. 11 Axial velocity profile along the centerline $(y=0, x=0)$ for the contraction-expansion geometry $\left(w_{\mathrm{c}}=26 \mu \mathrm{m}, h=55 \mu \mathrm{m}\right)$ as a function of the axial position $\left(-14<z / w_{\mathrm{c}}<0\right)$ for $R e=7.3$ : experimental (open symbols) and numerical (filled symbols)

contraction cannot be directly compared to those obtained in a simple expansion geometry, where fully developed flow in the upstream channel is usually imposed as an inlet condition (see, e.g. Drikakis 1997). In our case, the maximum velocity at the contraction exit is, in most cases, slightly below the actual fully developed value. As the flow rate increases, steady symmetric recirculations grow downstream of the expansion plane. This results in slower deceleration of material elements and a marked reduction in the magnitude of the extension rate $\left|\partial V_{z} / \partial z\right|$ along the centerline downstream of the expansion plane. By contrast the upstream profile remains almost unchanged with changes in flow rate and a maximum value given by $\left(\partial V_{z} /\right.$ $\partial z)_{\max } \approx 0.8\left\langle V_{z}\right\rangle_{\mathrm{c}} / w_{\mathrm{c}}$. This is an important result from a rheometric point of view; it allows the control of the (dimensional) strain rate by controlling the flow rate and then measuring the resulting pressure drop.

\section{Pressure drop}

The global pressure drop $\Delta P_{12}$ between the two pressure taps (located $3 \mathrm{~mm}$ upstream and downstream of the contraction plane) was measured experimentally using differential pressure sensors as discussed in Sect. 2.2. In Fig. 13 we compare experimental data for the global pressure drop with the corresponding numerical predictions. We examine the effect of inertia, by varying the flow rate in the range $0<Q \leq 12 \mathrm{ml} / \mathrm{h}$ (substituting for the relevant geometric quantities and the material properties of water we find $R e=7.28 Q$, with $Q$ in $\mathrm{ml} / \mathrm{h}$ ). The geometry, with $L_{\mathrm{c}}=100 \mu \mathrm{m}$ and $h=55 \mu \mathrm{m}$, was chosen to match the experimental conditions of Rodd et al. (2005). For low flow rates $(Q \leq 2 \mathrm{ml} / \mathrm{h})$, the pressure drop is seen to
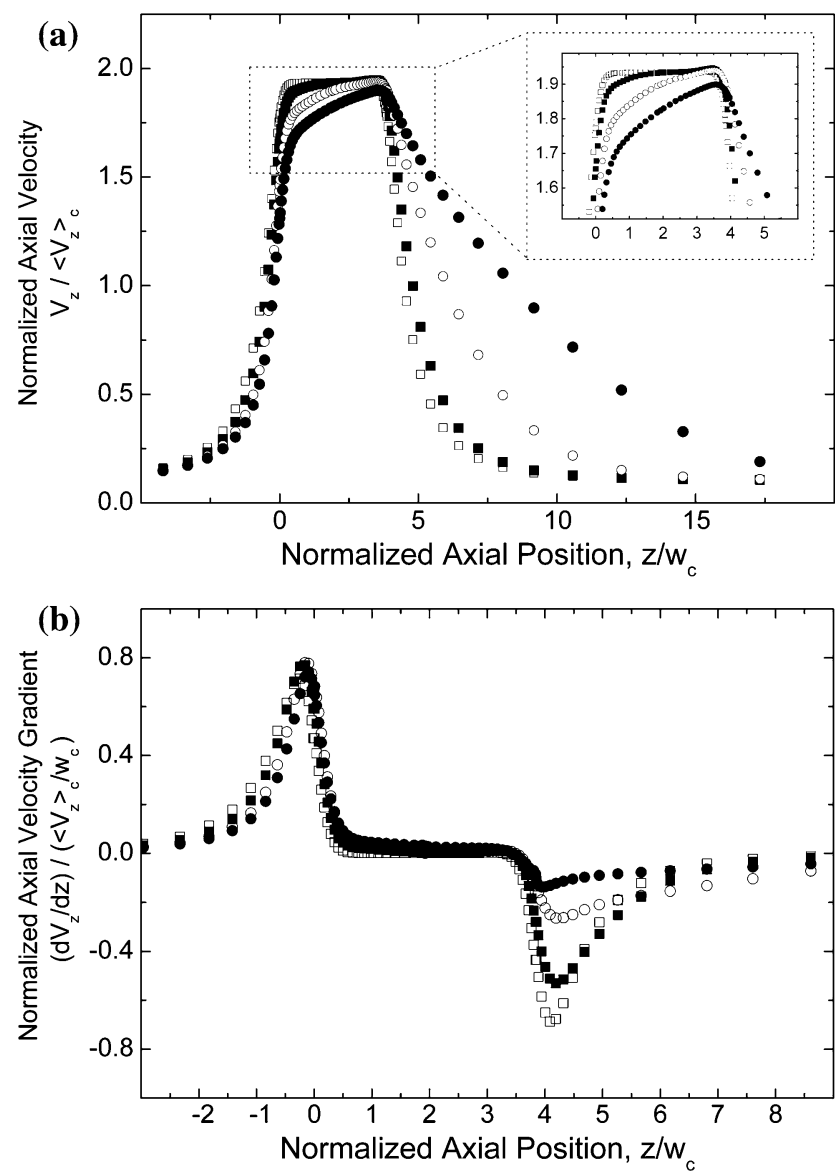

Fig. 12 Predicted axial velocity profiles (a) and corresponding strain rate profiles (b) along the centerline $(y=0, x=0)$ for $w_{\mathrm{c}}=26 \mu \mathrm{m}$, $h=55 \mu \mathrm{m}$ and $L_{\mathrm{c}}=100 \mu \mathrm{m}$ and a range of $R e: R e=0.73$ (open square), $R e=7.3$ (filled square), $R e=21.8$ (open circle) and Re $=43.7$ (filled circle)

increase linearly with $Q$ as expected. As the flow rate is further increased the non-linear convective terms in the momentum equation become important and the total pressure drop starts to deviate from the linear viscous scaling, as can be clearly observed in the $\log -\log$ inset on Fig. 13. Close agreement is observed between the numerical and experimental results over the whole range of experimental conditions.

Experimentally, it is a challenging task to obtain further details regarding the local evolution of the pressure field within the channel. Numerically, however, we can probe the pressure field in detail. In Fig. 14a, we show the evolution of the dimensionless pressure along the centerline $(y=0$ and $x=0)$ to illustrate the effect of fluid inertia. We also show for reference the scaled pressure expected for a hypothetical fully developed flow in all channels. This serves as a useful reference for comparisons. The effect of the contraction is clear, and is manifested in a drop in pressure just upstream of the entrance to the throat. This pressure drop is associated with the flow rearrangement 


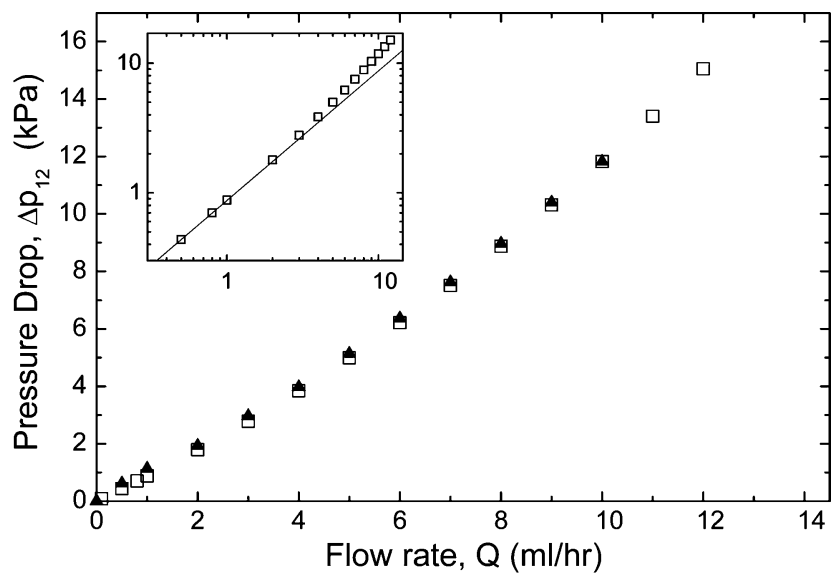

Fig. 13 Comparison of the total pressure drop determined experimentally (filled symbols) and numerically (open symbols) as a function of the flow rate. The inset shows the numerical results on a $\log -\log$ scale and compares the computed values with the linear behavior anticipated at low flow rates (solid line)

near the contraction plane and is the main source of difference between the computed profile and the hypothetical locally fully developed flow. There is also a small, but noticeable, exit effect near the expansion plane. The viscous flow in the narrow channel develops very rapidly at these flow rates. Varying the total length of the channel changes the total pressure drop across the device but does not influence the entrance correction appreciably (see Supplementary Information, Sect. S4). Figure 14b shows the longitudinal pressure gradient $\partial p / \partial z$ along the centerline for the contraction-expansion for a range of Reynolds numbers $(0.73 \leq R e \leq 43.7)$. There is an initial region of abrupt increase in the pressure gradient, from a situation of fully developed flow away from the contraction to a maximum at the contraction plane. As the Reynolds number increases, the increase in the normalized pressure gradient becomes more pronounced. Because of the reversibility of creeping flow, the exit region is a mirror image of the entrance region at low $R e$ and the maximum pressure gradient attained corresponds to that of a fully developed flow in the narrow channel. For higher flow rates, the pressure gradient beyond the contraction plane decays until it reaches a constant value consistent with Poiseuille flow in a channel of width $w_{\mathrm{c}}$ and depth $h$. Just upstream of the expansion plane, the pressure gradient starts to decrease once more, until it eventually becomes constant far downstream from the expansion at the value expected for viscous flow in a channel that is approximately 16 times wider than the throat. For the two highest values of $R e$, the pressure gradient actually changes sign before reaching this constant value as a result of the recirculating structures downstream of the expansion plane. To summarize these observations, the contraction entrance
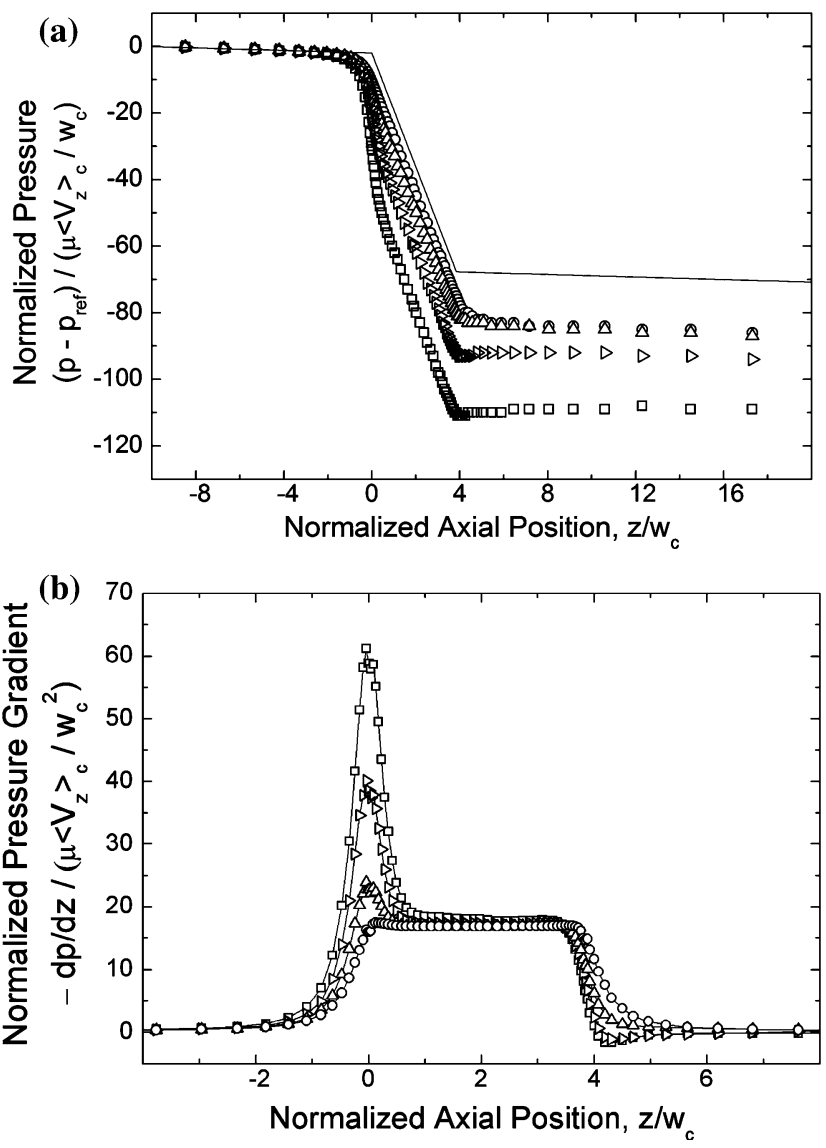

Fig. 14 Normalized pressure profiles (a) and pressure gradient (b) along the centerline $(y=0, x=0)$ for the 400:26:400 contractionexpansion geometry with $h=55 \mu \mathrm{m}$ and $L_{\mathrm{c}}=100 \mu \mathrm{m}: R e=0.73$ (open circle), $R e=7.3$ (open triangle), $R e=21.8$ (tilted triangle) and $R e=43.7$ (open square); the solid line in (a) corresponds to a hypothetical pressure profile if there were no entrance or exit effects and the flow was locally fully developed at all times

region is responsible for a large fraction of the extra pressure drop, and this increases substantially with flow inertia.

We now proceed to focus on the extra (or excess) pressure drop $\Delta p_{\text {exc }}$ associated with the contractionexpansion flow. This cannot be readily measured in experiments but can be evaluated from measurements of the total pressure drop and application of an appropriate analytic decomposition (Boger 1987; Kang et al. 2006). Numerical computations of the full pressure field can be used to explore the validity of the protocols employed in processing the experimental data.

The excess pressure drop is calculated by subtracting from the global pressure drop the net pressure drop associated with the fully developed Poiseuille flow in the upstream and downstream parts of the channel, plus that in the narrow contraction: 
$\Delta p_{\mathrm{exc}}=\Delta p_{12}-\left(\frac{\mathrm{d} p}{\mathrm{~d} z}\right)_{\mathrm{u}} L_{\mathrm{u}}-\left(\frac{d \mathrm{p}}{d \mathrm{z}}\right)_{\mathrm{d}} L_{\mathrm{d}}-\left(\frac{\mathrm{d} p}{\mathrm{~d} z}\right)_{\mathrm{c}} L_{\mathrm{c}}$

where the subscript " $\mathrm{u}$ " refers to the channel upstream of the contraction plane, "d" to the channel downstream of the expansion plane, and "c" to the narrow channel in the contraction region. An alternative dimensionless quantity commonly reported is the Couette correction defined as $C=\Delta P_{\text {exc }} / 2 \tau_{\mathrm{w}}$ (Boger 1982), i.e. as the excess pressure drop arising from the sudden contraction and expansion scaled with $2 \tau_{\mathrm{w}}$, where $\tau_{\mathrm{w}}$ is the average wall shear stress of the fully developed flow in the contraction channel:

$2 \tau_{\mathrm{w}}=-(\mathrm{d} p / \mathrm{d} z)_{\mathrm{c}} D_{\mathrm{h}, \mathrm{c}} / 2$

The effect of fluid inertia on the excess pressure drop and corresponding Couette correction are presented in Fig. 15. The excess pressure drop arises mainly from the entrance effects described in our analysis of Fig. 14a. These entry effects become more pronounced at higher flow rates, and thus the excess pressure drop and Couette correction increase substantially with $R e$. For the range of contraction lengths tested, $L_{\mathrm{c}}$ does not seem to have an effect on the excess pressure drop, provided that the flow has sufficient residence time for the pressure gradient to fully develop and that there are portions of the narrow contraction where the entrance and exit effects are not felt. This is achieved for the range of $R e$ used, except for the case of the contraction with length $L_{\mathrm{c}}=100 \mu \mathrm{m}$ and for the higher Reynolds numbers.

The Bagley analysis and associated Bagley plot is a common tool in the polymer processing field in which axisymmetric capillaries are generally used to measure entrance effects in complex fluids (Kim and Dealy 2001). The Bagley plot is a graph of the total pressure drop versus the length to diameter ratio of the die, with apparent wall

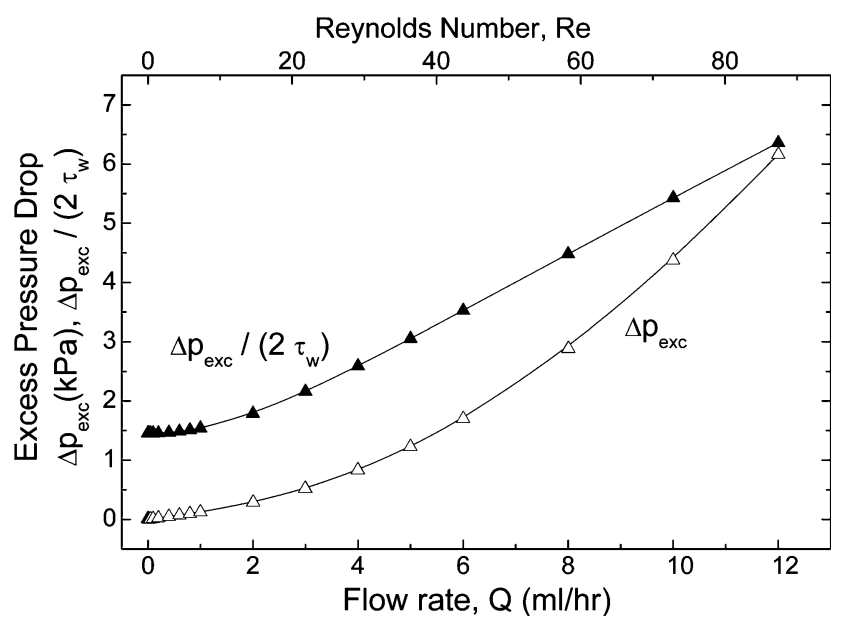

Fig. 15 Effect of inertia on the excess pressure drop for a contraction-expansion geometry with $w_{\mathrm{c}}=26 \mu \mathrm{m}, h=55 \mu \mathrm{m}$ and $L_{\mathrm{c}}=400 \mu \mathrm{m}$ shear rate as the control parameter (Kim and Dealy 2001). The measured data falls on straight lines each corresponding to a specific value of the apparent shear rate and extrapolation to the limit of zero capillary length gives the excess pressure drop. Typically for axisymmetric geometries, the total pressure drop is calculated between two points, one at a fixed upstream location $\left(z=-z_{0}\right)$ and the other on the free jet exiting downstream (atmospheric pressure), for several different $L_{\mathrm{c}}$ keeping the flow rate constant. The extrapolation of the fitted straight line to $L_{\mathrm{c}}=0$ allows the calculation of the Bagley excess pressure drop and corresponding dimensionless Bagley correction (Mitsoulis et al. 1998). This method yields good results in axisymmetric contractions with high contraction ratios, since the pressure drop upstream of the contraction due to fully developed flow as well as at the exit of the capillary are negligible compared to the excess pressure drop. In the case of microfluidic devices, where the channels are typically planar with a constant out-of-plane dimension (i.e. depth) smaller than the upstream channel width, the effective contraction ratio-based on hydraulic diameters $\left(D_{\mathrm{h}, \mathrm{u}} / D_{\mathrm{h}, \mathrm{c}}\right)$-is much smaller when compared at an equivalent nominal contraction ratio $w_{\mathrm{u}} / w_{\mathrm{c}}$ as in the axisymmetric case. In such cases, depending on the positions $z_{0}$ chosen for the pressure transducers we would obtain different values of $\Delta P_{\text {exc }}$. It is thus helpful to rethink the Bagley analysis for microfluidic devices. To determine the equivalent to the Bagley correction for such planar geometries, the total pressure drop was calculated between pressure taps located at positions that are proportional to the length of the contraction. For example, the total pressure drop for the Bagley plot, $\Delta P_{\text {Bagley, }}$ for the contractionexpansion device should be calculated between:

- $z=-\alpha$ and $z=L_{\mathrm{c}}+\beta$, for $L_{\mathrm{c}}=100 \mu \mathrm{m}$,

- $z=-2 \alpha$ and $z=L_{\mathrm{c}}+2 \beta$, for $L_{\mathrm{c}}=200 \mu \mathrm{m}$,

- $z=-4 \alpha$ and $z=L_{\mathrm{c}}+4 \beta$, for $L_{\mathrm{c}}=400 \mu \mathrm{m}$.

for the contraction-expansions simulated in the present work, i.e. $L_{\mathrm{c}}=100,200$ and $400 \mu \mathrm{m}$. Extrapolation to the limiting case $L_{\mathrm{c}}=0$ of the straight line fitted to the total pressure drop will yield the relevant Bagley correction. Using this construction, the calculated $\Delta P_{\mathrm{exc}}$ will be the same, whatever $\alpha$ and $\beta$ values we decide to use, provided that they are proportional to the contraction length and that they are sufficiently separated from the contraction so that there are no exit or entrance effects. In the calculations, we have used arbitrarily the values $\alpha=\beta=1 \mathrm{~mm}$.

Figure 16 shows the Bagley plot corresponding to our contraction-expansion geometry for various Reynolds numbers in the range from $R e=0$ (creeping flow) to $R e=87.4$. We used three different lengths of the contraction: 100, 200 and $400 \mu \mathrm{m}$, and since we are dealing with a planar geometry, we report the ratio of $L_{\mathrm{c}}$ to the 


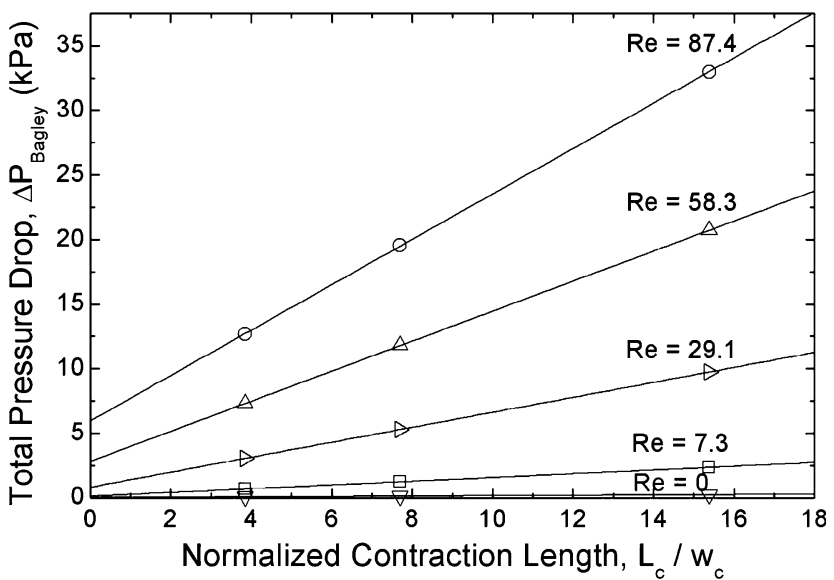

Fig. 16 Bagley plot for a range of Reynolds numbers $(R e \leq 87.4)$ in the 400:26:400 contraction-expansion geometry with $h=55 \mu \mathrm{m}$

width of the contraction channel, $w_{\mathrm{c}}$. As expected, for the lower flow rates, the total pressure drop increases linearly as a function of the length of the contraction. For the two highest $R e$, there is a slight deviation from linearity, which is related to the fact that for the shorter contraction the residence time of material elements in the narrow channel is not sufficient for the flow to develop fully. This will have an effect on the accuracy of the Bagley prediction of excess pressure drop. The Bagley analysis results in the same values as the corresponding Couette correction, except for the highest Reynolds numbers $(R e>50)$, for which the Bagley analysis yields slightly lower values. If we calculate the Bagley correction for the highest Reynolds numbers ( $R e=58.3$ and $R e=87.4)$ using only on the data corresponding to $L_{\mathrm{c}}=200$ and $400 \mu \mathrm{m}$ (where the flow is fully developed in the middle of the smaller channel), then we achieve perfect agreement between both methods. This is an important finding, since the Bagley method of determining the excess pressure drop is likely to be more amenable for use in an experimental context.

As in the previous section, we have also studied the effect of the aspect ratio AR on the pressure drop for a range of Reynolds number, and this is shown in Fig. 17. The Couette correction, calculated using Eqs. (5) and (6), increases with the Reynolds number for large values of $h$, but approaches a constant value independent of $R e$ as the channel depth decreases; however, this value depends on $h$ itself. Better insight is obtained if instead we plot a modified Couette correction, $\quad C^{*}$, defined as $C *=\frac{\Delta P_{\text {exc }}}{2 \tau_{\mathrm{w}}^{*}}=$ $\frac{\Delta P_{\mathrm{exc}}}{-(\mathrm{d} p / \mathrm{d} z)_{\text {contraction }} \times W_{\mathrm{c}}}$, as a function of the Reynolds number based on channel width $\left(R e_{\mathrm{w}_{\mathrm{c}}}\right)$. This parameter represents the extra pressure drop due to the contraction-expansion in terms of the number of equivalent multiples of the (small) microchannel widths that would produce the same pressure loss. The meaning of this parameter can be more easily
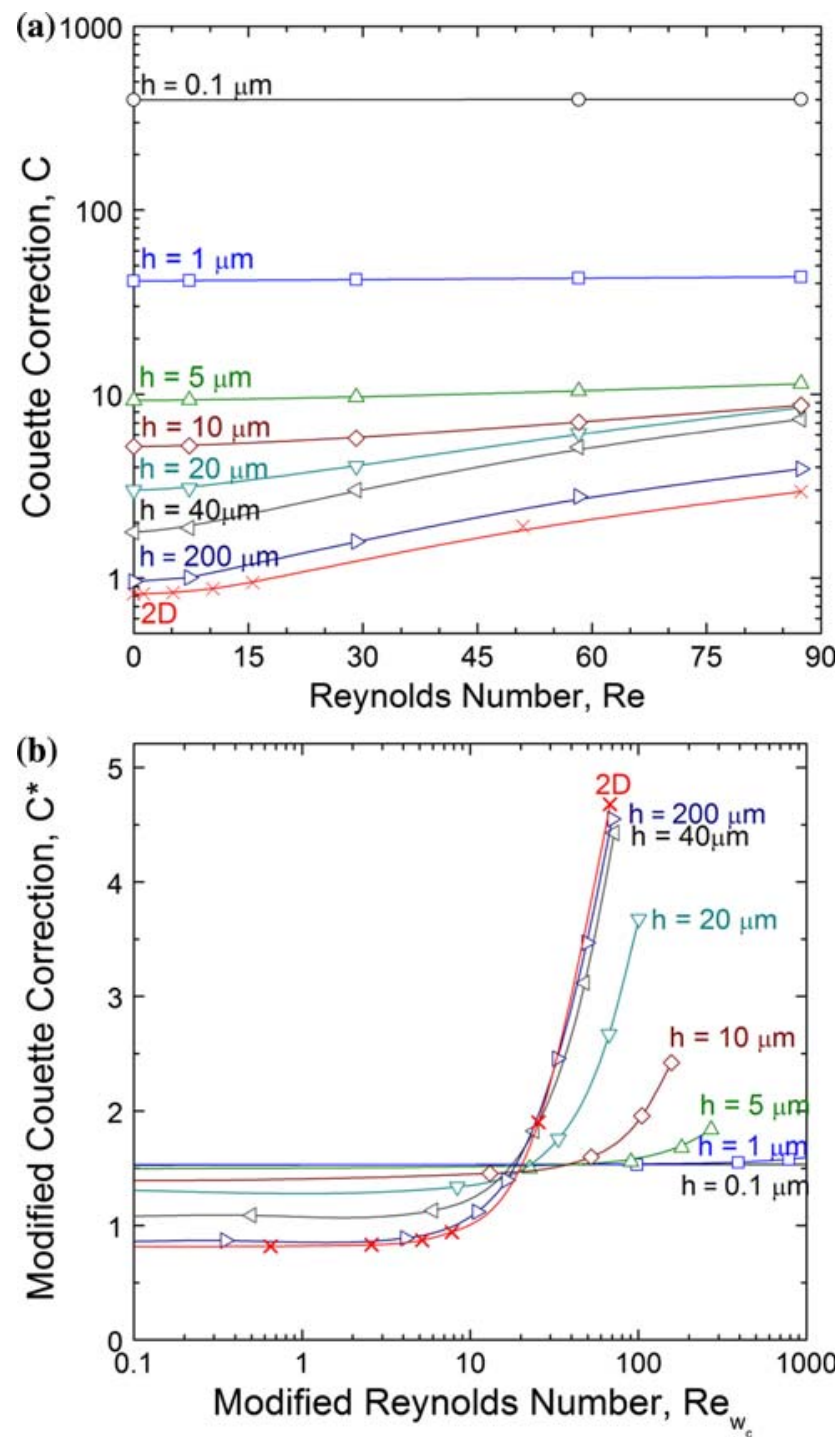

Fig. 17 Effect of the aspect ratio on the standard (a) and modified Couette correction (b) for the 400:26:400 contraction-expansion geometry: $\quad h=0.1 \mu \mathrm{m} \quad\left(\mathrm{AR}=3.85 \times 10^{-3}\right) ; \quad h=1 \mu \mathrm{m}$ $\left(\mathrm{AR}=3.85 \times 10^{-2}\right) ; h=5 \mu \mathrm{m}\left(\mathrm{AR}=1.92 \times 10^{-1}\right) ; h=10 \mu \mathrm{m}$ $\left(\mathrm{AR}=3.85 \times 10^{-1}\right) ; h=20 \mu \mathrm{m}\left(\mathrm{AR}=7.69 \times 10^{-1}\right) ; h=40 \mu \mathrm{m}$ $(\mathrm{AR}=1.54) ; h=200 \mu \mathrm{m}(\mathrm{AR}=7.69) ; 2 \mathrm{D}(\mathrm{AR} \rightarrow \infty)$

understood by analogy with the pressure drops reported in turbulent flows that arise from minor losses in pipe systems. These are commonly expressed as a loss factor $K$ which can also be related to the number of equivalent pipe diameters. The asymptotic behavior of the excess pressure drop is now much clearer: the modified Couette correction is bounded between two limiting cases corresponding to Hele-Shaw flow and 2D flow. For a 15.4:1:15.4 contraction expansion at low $R e_{\mathrm{w}_{\mathrm{c}}}$, the results are bounded between constant values of 0.84 (2D flow, AR $\rightarrow \infty$ ) and 1.52 (Hele-Shaw flow, AR $\rightarrow 0$ ). For high aspect ratios, the modified Couette correction increases substantially with increasing inertia beyond $R e_{\mathrm{w}_{\mathrm{c}}} \approx 5$, as illustrated in Fig. 17b. 


\section{Conclusions}

A series of numerical simulations have been performed for Newtonian fluid flow through an abrupt contractionexpansion microfluidic device over a range of aspect ratios and Reynolds numbers. The numerical results are in good agreement with experimental measurements of the kinematics and pressure drop for the same conditions (fluid and geometry). The numerical calculations allow for a more detailed analysis of the flow and a broader range of conditions than the experiments, thus complementing the experimental work and guiding future device design.

It was shown that although the flow may appear to be quasi-2D, in fact, for typical values of the aspect ratio that can be obtained experimentally, it is highly three-dimensional with open spiraling recirculating structures in the stagnant corner regions. For the range of $R e$ values which can be achieved experimentally in our set-up $(R e \lesssim 90$, $\mathrm{AR}=2.1$ ), computations and observation show that the flow always remains symmetric about the center plane. As inertial effects become important, the formation and growth of lip recirculations is observed downstream of the expansion. At $R e \approx 20$, the lip recirculations reach the outer walls of the expansion geometry and grow downstream. Beyond this transition, the recirculation size increases monotonically with $R e$. Two-dimensional simulations were also carried out in a corresponding idealized channel (corresponding to $A R>>1$ ) in which the effects of the upper and lower endwalls are negligible. A supercritical bifurcation is found in 2D flow for a critical Reynolds number of $R e_{(2 \mathrm{D})} \approx 28$. The flow asymmetries become more pronounced with increasing Reynolds number, and large stable recirculations are formed downstream of the expansion. This work illustrates that even relatively simple planar contraction-expansion geometries can lead to highly complex 3D flows. Simpler 2D approximations can lead to large quantitative errors in the predictions of measurable quantities such as recirculation size and excess pressure drop which may not even be qualitatively acceptable.

The dimensionless excess pressure drop associated with the converging-diverging flow was evaluated using both the Couette and Bagley approaches. We suggest a modified means of calculating the Bagley correction which appears to be more appropriate for planar microchannels with a contraction followed by an expansion. In this way, the Couette and Bagley correction methods yield consistent results provided that fully developed flow is attained at some point in the contraction region. The excess pressure drop is caused primarily by the flow rearrangement in the entrance region, which becomes more pronounced as the Reynolds number is increased. The effect of varying the device aspect ratio (AR) on the excess pressure drop has also been studied in detail. Analysis of both the computed velocity and pressure fields show that the results are bounded between two well-defined limiting cases: that of $2 \mathrm{D}$ viscous flow $(\mathrm{AR} \rightarrow \infty)$ and viscous potential flow in the limiting case of Hele-Shaw flow (AR $\rightarrow 0$ ).

The ability of the $3 \mathrm{D}$ numerical calculations to accurately capture both the kinematics and dynamics observed experimentally provides a mechanism for rapidly exploring the consequences of changes to the geometric parameters governing the flow in a microfluidic device. This computational rheometric capability will prove useful in optimizing the design of future microfluidic rheometers.

Acknowledgement M. S. N. Oliveira would like to thank Fundação para a Ciência e a Tecnologia (FCT), Portugal, for financial support (SFRH/BPD/15005/2004, SFRH/BPD/34141/2006). M. S. N. Oliveira and M. A. Alves acknowledge the financial support provided by FCT and FEDER under program POCI 2010 (projects PPTDC/EME/ 59338/2004 and POCI/EQU/59256/2004).

\section{References}

Alves MA, Pinho FT, Oliveira PJ (2000) Effect of a high-resolution differencing scheme on finite-volume predictions of viscoelastic flows. J Non-Newt Fluid Mech 93:287-314

Alves MA, Oliveira PJ, Pinho FT (2003) A convergent and universally bounded interpolation scheme for the treatment of advection. Int J Num Meth Fluids 41:47-75

Alves MA, Oliveira PJ, Pinho FT (2004) On the effect of contraction ratio in viscoelastic flow through abrupt contractions. J NonNewt Fluid Mech 122:117-130

Alves MA, Pinho FT, Oliveira PJ (2005) Visualizations of Boger fluid flows in a 4:1 square-square contraction. AIChE J 51:2908-2922

Alves MA, Poole RJ (2007) Divergent flow in contractions. J NonNewt Fluid Mech 144:141-148

Bagley EB (1957) End corrections in the capillary flow of polyethylene. J Appl Phys 28:624-627

Barnes HA, Hutton JF, Walters K (1989) An introduction to rheology. Elsevier: Distributors for the U.S. and Canada, Elsevier Science Pub. Co., Amsterdam, New York

Barrat JL, Bocquet L (1999) Large slip effect at a nonwetting fluidsolid interface. Phys Rev Lett 82:4671-4674

Battaglia F, Tavener SJ, Kulkarni AK, Merkle CL (1997) Bifurcation of low Reynolds number flows in symmetric channels. AIAA J 35:99-105

Bayraktar T, Pidugu SB (2006) Characterization of liquid flows in microfluidic systems. Int J Heat Mass Transf 49:815-824

Binding DM (1988) An approximate analysis for contraction and converging flows. J Non-Newt Fluid Mech 27:173-189

Binding DM, Couch MA, Walters K (1998) The pressure dependence of the shear and elongational properties of polymer melts. J NonNewt Fluid Mech 79:137-155

Boger DV (1982) Circular entry flows of inelastic and viscoelastic fluids. In: Mujumdar AS, Mashelkar RA (eds) Advances of transport processes, vol 2. Wiley Eastern, New Delhi, pp 43-98

Boger DV (1987) Viscoelastic flows through contractions. Annu Rev Fluid Mech 19:157-182

Boger DV, Binnington RJ (1990) Circular entry flows of fluid M1. J Non-Newt Fluid Mech 35:339-360

Brown RA, McKinley GH (1994) Report on the VIIIth Int. workshop on numerical methods in viscoelastic flows. J Non-Newt Fluid Mech 52:407-413 
Cherdron W, Durst F, Whitelaw JH (1978) Asymmetric flows and instabilities in symmetric ducts with sudden expansions. J Fluid Mech 84:13-31

Chiang TP, Sheu TWH, Wang SK (2000) Side wall effects on the structure of laminar flow over a plane-symmetric sudden expansion. Comp Fluids 29:467-492

Cogswell FN (1972a) Converging flow of polymer melts in extrusion dies. Polymer Eng Sci 12:64-73

Cogswell FN (1972b) Measuring extensional rheology of polymer melts. Trans Soc Rheol 16:383-403

Drikakis D (1997) Bifurcation phenomena in incompressible sudden expansion flows. Phys Fluids 9:76-87

Durst F, Melling A, Whitelaw JH (1974) Low Reynolds-number flow over a plane symmetric sudden expansion. J Fluid Mech 64:111128

Fearn RM, Mullin T, Cliffe KA (1990) Nonlinear flow phenomena in a symmetric sudden expansion. J Fluid Mech 211:595-608

Gad-el-Hak M (2002) The MEMS handbook. CRC Press, Boca Raton

Hassager O (1988) Working group on numerical techniques (Vth workshop on numerical methods in non-Newtonian flow). J NonNewt Fluid Mech 29:2-5

Hawa T, Rusak Z (2001) The dynamics of a laminar flow in a symmetric channel with a sudden expansion. J Fluid Mech 436:283-320

James DF, Chandler GM, Armour SJ (1990) A converging channel rheometer for the measurement of extensional viscosity. J NonNewt Fluid Mech 35:421-443

Kang K, Lee LJ, Koelling KW (2005) High shear microfluidics and its application in rheological measurement. Exp Fluids 38: 222-232

Kang K, Koelling KW, Lee LJ (2006) Microdevice end pressure evaluations with Bagley correction. Microfluid Nanofluid 2:223235

Karniadakis G, Beskok A, Aluru NR (2005) Microflows and nanoflows: fundamentals and simulation. Springer, New York

Kim S, Dealy JM (2001) Design of an orifice die to measure entrance pressure drop. J Rheol 45:1413-1419

Lauga E, Stroock AD, Stone HA (2004) Three-dimensional flows in slowly varying planar geometries. Phys Fluids 16:3051-3062

Lee WY, Wong M, Zohar Y (2002) Microchannels in series connected via a contraction/expansion section. J Fluid Mech 459:187-206

Leonard BP (1979) Stable and accurate convective modeling procedure based on quadratic upstream interpolation. Comp Meth Appl Mech Eng 19:59-98

McDonald JC, Duffy DC, Anderson JR, Chiu DT, Wu HK, Schueller OJA, Whitesides GM (2000) Fabrication of microfluidic systems in poly(dimethylsiloxane). Electrophoresis 21:27-40

Mishra S, Jayaraman K (2002) Asymmetric flows in planar symmetric channels with large expansion ratio. Int J Num Meth Fluids 38:945-962

Mitsoulis E, Hatzikiriakos SG, Christodoulou K, Vlassopoulos D (1998) Sensitivity analysis of the Bagley correction to shear and extensional rheology. Rheol Acta 37:438-448

Moffatt HK (1964) Viscous and resistive eddies near a sharp corner. J Fluid Mech 18:1-18

Neto C, Evans DR, Bonaccurso E, Butt HJ, Craig VSJ (2005) Boundary slip in Newtonian liquids: a review of experimental studies. Rep Prog Phys 68:2859-2897
Oliveira MSN, Alves MA, McKinley GH, Pinho FT (2007a) Viscous flow through microfabricated hyperbolic contractions. Exp Fluids 43:437-451

Oliveira MSN, Oliveira PJ, Pinho FT, Alves MA (2007b) Effect of contraction ratio upon viscoelastic flow in contractions: the axisymmetric case. J Non-Newt Fluid Mech 147:92-108

Oliveira MSN, Yeh R, McKinley GH (2006) Iterated stretching, extensional rheology and formation of beads-on-a-string structures in polymer solutions. J Non-Newt Fluid Mech 137:137-148

Oliveira PJ (2003) Asymmetric flows of viscoelastic fluids in symmetric planar expansion geometries. J Non-Newt Fluid Mech 114:33-63

Oliveira PJ, Pinho FT (1999) Numerical procedure for the computation of fluid flow with arbitrary stress-strain relationships. Num Heat Transf B 35:295-315

Oliveira PJ, Pinho FT, Pinto GA (1998) Numerical simulation of nonlinear elastic flows with a general collocated finite-volume method. J Non-Newt Fluid Mech 79:1-43

Owens RG, Phillips NT (2002) Computational rheology. Imperial College Press, London

Pit R, Hervet H, Leger L (2000) Direct experimental evidence of slip in hexadecane: solid interfaces. Phys Rev Lett 85:980-983

Revuelta A (2005) On the two-dimensional flow in a sudden expansion with large expansion ratios. Phys Fluids 17:028102 1-4

Rodd LE, Scott TP, Boger DV, Cooper-White JJ, McKinley GH (2005) The inertio-elastic planar entry flow of low-viscosity elastic fluids in micro-fabricated geometries. J Non-Newt Fluid Mech 129:1-22

Rothstein JP, McKinley GH (2001) The axisymmetric contractionexpansion: the role of extensional rheology on vortex growth dynamics and the enhanced pressure drop. J Non-Newt Fluid Mech 98:33-63

Shapira M, Degani D, Weihs D (1990) Stability and existence of multiple solutions for viscous-flow in suddenly enlarged channels. Comp Fluids 18:239-258

Sobey IJ, Drazin PG (1986) Bifurcations of two-dimensional channel flows. J Fluid Mech 171:263-287

Townsend P, Walters K (1994) Expansion flows of non-Newtonian liquids. Chem Eng Sci 49:749-763

Tsai C-H, Chen H-T, Wang Y-N, Lin C-H, Fu L-M (2006) Capabilities and limitations of 2-dimensional and 3-dimensional numerical methods in modeling the fluid flow in sudden expansion microchannels. Microfluid Nanofluid 3:13-18

Tuladhar TR, Mackley MR (2008) Filament stretching rheometry and break-up behaviour of low viscosity polymer solutions and inkjet fluids. J Non-Newt Fluid Mech 148:97-108. doi:10.1016/ j.jnnfm.2007.04.015

Wereley ST, Meinhart CD (2004) Micron-resolution particle image velocimetry. In: Breuer KS (ed) Microscale diagnostic techniques. Springer, Berlin

White SA, Gotsis AD, Baird DG (1987) Review of the entry flow problem-experimental and numerical. J Non-Newt Fluid Mech 24:121-160

Whitesides GM (2006) The origins and the future of microfluidics. Nature 442:368-373

Whitesides GM, Stroock AD (2001) Flexible methods for microfluidics. Phys Today 54:42-48

Wille R, Fernholz H (1965) Report on first European mechanics colloquium on Coanda effect. J Fluid Mech 23:801-819 